University of Tennessee Health Science Center

UTHSC Digital Commons

\title{
$5-2009$
}

\section{Immediate Effect of Complete Denture Occlusal Errors on Masticatory Muscle EMG Activity in Denture Wearers: A Pilot Study}

Swati Ahuja

University of Tennessee Health Science Center

Follow this and additional works at: https://dc.uthsc.edu/dissertations

Part of the Prosthodontics and Prosthodontology Commons

\section{Recommended Citation}

Ahuja, Swati , "Immediate Effect of Complete Denture Occlusal Errors on Masticatory Muscle EMG Activity in Denture Wearers: A Pilot Study" (2009). Theses and Dissertations (ETD). Paper 13. http://dx.doi.org/ 10.21007/etd.cghs.2009.0009. 


\title{
Immediate Effect of Complete Denture Occlusal Errors on Masticatory Muscle EMG Activity in Denture Wearers: A Pilot Study
}

\begin{abstract}
In the last three decades, little scientific progress has been seen in relation to the occlusal aspects in complete dentures. Equal distribution of loading forces to the edentulous denture foundation is an important factor affecting stability, patient comfort and acceptance of complete dentures. Also, understanding the functional behavior of masticatory muscles for complete denture wearers is important for diagnosing and planning the clinical treatment.

The purpose of the present study was to determine the effect of unilateral and bilateral occlusal interferences on the masticatory muscle activity in edentulous patients, and how patients respond to occlusal interferences both physiologically and psychologically over a brief period of time.

In ten subjects rigid bilateral intercuspal interferences were placed on the most distal teeth of the mandibular complete denture. Subjects were asked to clench maximally and the surface EMG of the right and the left masseter and the temporalis muscles were recorded. Bilateral interferences were removed and the subjects were divided into two groups. Group A received unilateral right interference and group B received unilateral left interference. Surface EMG of the right and the left masseter and the temporalis muscles was recorded at maximal clench. Occlusal interference was removed for both the groups and final EMG recordings were made at maximal clench for all patients. Patients responded to questionnaires after each intervention and finally after removal of occlusalinterference.

Student's paired T test was used to analyze the data. Results demonstrated no significant difference in EMG values of the patients at baseline and after the introduction of bilateral interferences. There was no significant difference in the EMG values of patients at baseline and after the introduction of unilateral right interferences. In the 5 patients with unilateral left occlusal interferences there was a significant reduction in the EMG activity of the left masseter. There was also no significant difference in the EMG values at baseline and after the removal of occlusal interferences. The analysis of the questionnaires did not reveal any significant finding.
\end{abstract}

\section{Document Type}

Thesis

Degree Name

Master of Dental Science (MDS)

Program

Prosthodontics

Research Advisor

Robert Brandt D.D.S, M.S.

\section{Keywords}

Complete denture, EMG, Muscle activity, Occlusion, T Scan

\section{Subject Categories}

Dentistry | Medicine and Health Sciences | Prosthodontics and Prosthodontology 


\title{
IMMEDIATE EFFECT OF COMPLETE DENTURE OCCLUSAL ERRORS ON MASTICATORY MUSCLE EMG ACTIVITY IN DENTURE WEARERS: A PILOT STUDY
}

\author{
A Thesis \\ Presented for \\ The Graduate Studies Council \\ The University of Tennessee \\ Health Science Center
}

\author{
In Partial Fulfillment \\ Of the Requirements for the Degree \\ Master of Dental Science \\ From The University of Tennessee
}

By

Swati Ahuja, B.D.S.

May 2009 
Copyright (C) 2009 by Swati Ahuja All rights reserved 


\section{ACKNOWLEDGEMENTS}

I would like to express my gratitude to everyone who has helped me in my endeavor of pursuing a Master of Dental Science in Prosthodontics. To Dr. David Cagna, without his immense knowledge and hard work, this research project would not have been possible. To my committee members, Drs. Robert Brandt, Russell Wicks and Mark Scarbecz for their advice and experience. I also want to thank Bioresearch and Teskscan for their generous donations. Lastly, I must thank my family for their love and support. 


\begin{abstract}
In the last three decades, little scientific progress has been seen in relation to the occlusal aspects in complete dentures. Equal distribution of loading forces to the edentulous denture foundation is an important factor affecting stability, subject comfort and acceptance of complete dentures. Also, understanding the functional behavior of masticatory muscles for complete denture wearers is important for diagnosing and planning the clinical treatment.
\end{abstract}

The purpose of the present study was to determine the effect of unilateral and bilateral occlusal interferences on the masticatory muscle activity in edentulous subjects, and how subjects respond to occlusal interferences both physiologically and psychologically over a brief period of time.

In ten subjects rigid bilateral intercuspal interferences were placed on the most distal teeth of the mandibular complete denture. Subjects were asked to clench maximally and the surface EMG of the right and the left masseter and the temporalis muscles were recorded. Bilateral interferences were removed and the subjects were divided into two groups. Group A received unilateral right interference and group B received unilateral left interference. Surface EMG of the right and the left masseter and the temporalis muscles was recorded at maximal clench. Occlusal interference was removed for both the groups and final EMG recordings were made at maximal clench for all subjects. Subjects responded to questionnaires after each intervention and finally after removal of occlusal interference.

Student's paired T-test was used to analyze the data. Results demonstrated no significant difference in EMG values of the subjects at baseline and after the introduction of bilateral interferences. There was no significant difference in the EMG values of subjects at baseline and after the introduction of unilateral right interferences. In the 5 subjects with unilateral left occlusal interferences there was a significant reduction in the EMG activity of the left masseter. There was also no significant difference in the EMG values at baseline and after the removal of occlusal interferences. The analysis of the questionnaires did not reveal any significant finding. 


\section{TABLE OF CONTENTS}

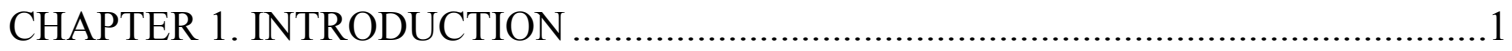

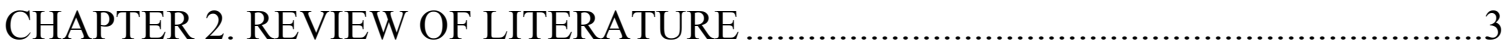

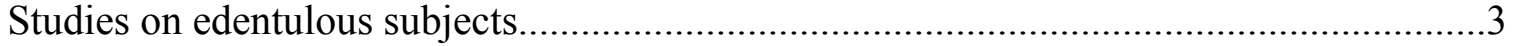

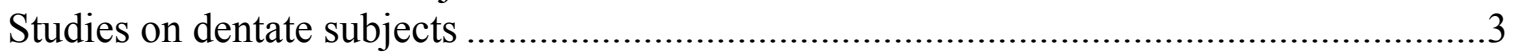

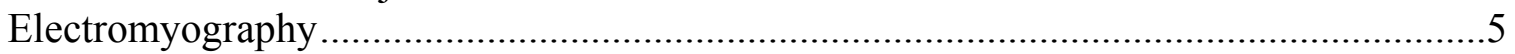

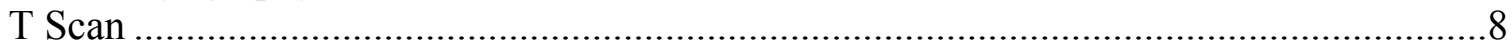

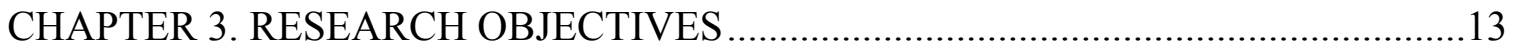

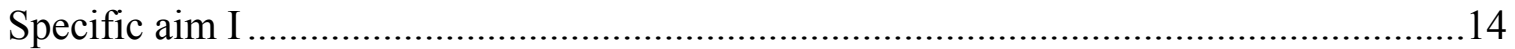

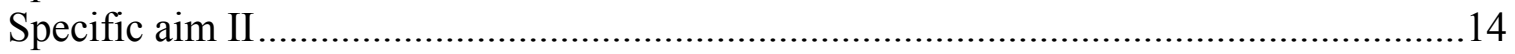

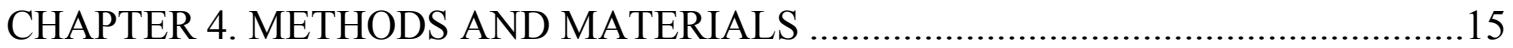

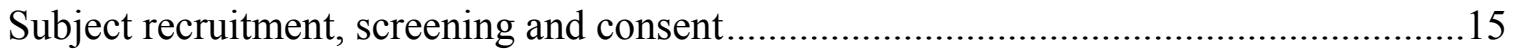

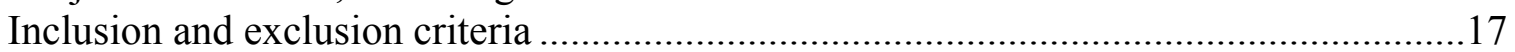

Characteristics of the subject population ................................................................ 17

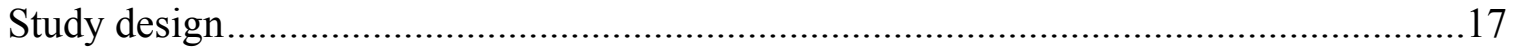

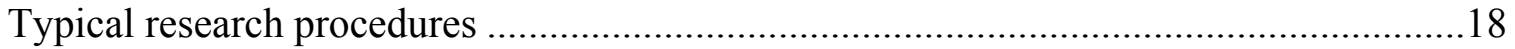

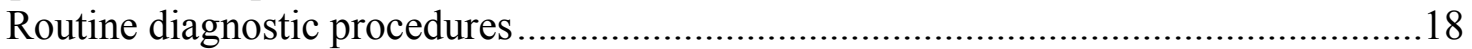

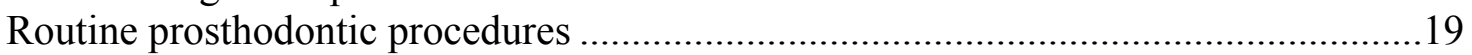

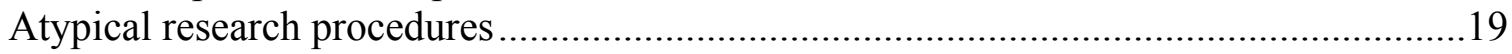

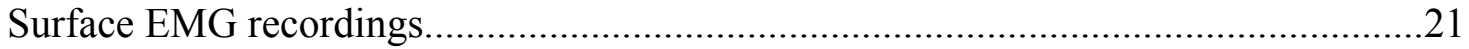

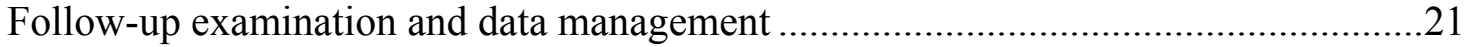

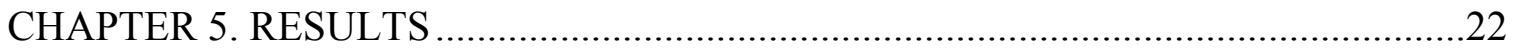

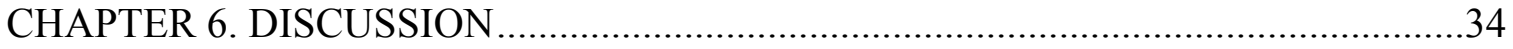

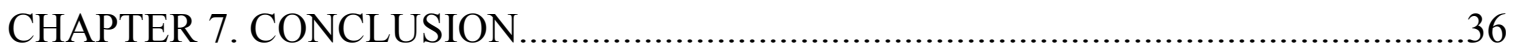

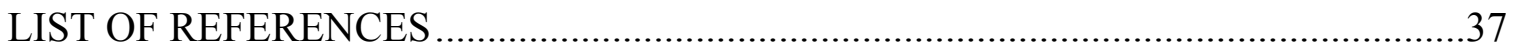

APPENDIX A. QUESTIONS AND EXAMPLE OF RESPONSE CHOICES FOR SUBJECT ASSESMENT OF STUDY DENTURES AFTER THE INTRODUCTION OF OCCLUSAL INTERFERENCES .........................................40

APPENDIX B. QUESTIONS AND EXAMPLE OF RESPONSE CHOICES FOR SUBJECT ASSESMENT OF STUDY DENTURES AFTER THE ELIMINATION

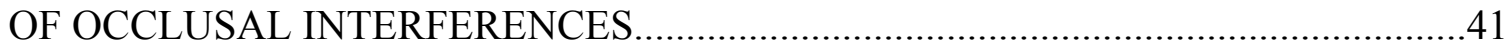

VITA 


\section{LIST OF FIGURES}

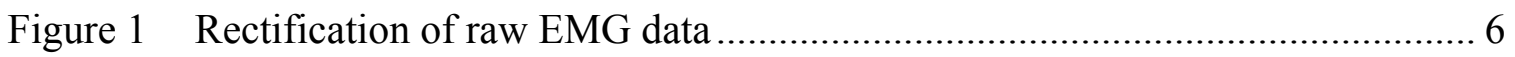

Figure 2 Application of averaging filter to the rectified EMG data............................... 7

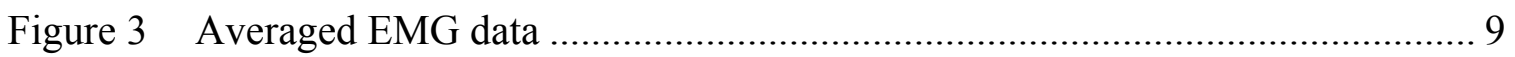

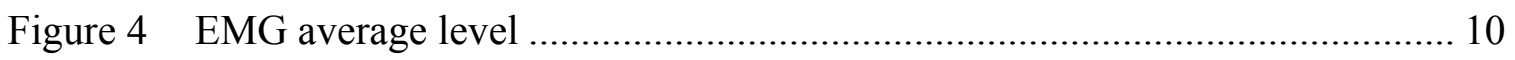

Figure 5 Presentation of EMG data on the computer screen...................................... 11

Figure 6 Presentation of T Scan data on the computer screen .................................... 12

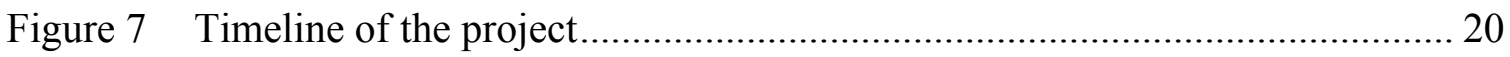

Figure 8 Timeline and the graphical analysis of the EMG activity at baseline (To) and EMG activity after the introduction of the bilateral interferences (Bil) in five subjects who would receive right sided unilateral interference........... 25

Figure 9 Timeline and the graphical analysis of the EMG activity at baseline (T0) and EMG activity after the introduction of the bilateral interferences (Bil) in five subjects who would receive left sided unilateral interference.

Figure 10 Timeline and the graphical analysis of the EMG activity at baseline (T0) and EMG activity after the introduction of the bilateral interferences (Bil) in all subjects

Figure 11 Timeline and the graphical analysis of the EMG activity at baseline (T0) and EMG activity after the introduction of the right interferences (Unil_R) in five subjects...

Figure 12 Timeline and the graphical analysis of the EMG activity at baseline (T0) and EMG activity after the introduction of the left interferences (Unil_L) in five subjects

Figure 13 Timeline and the graphical analysis of the EMG activity at baseline (T0) and EMG activity after the elimination of the interferences (Corrected) of five subjects who received right sided unilateral interference.

Figure 14 Timeline and the graphical analysis of the EMG activity at baseline (T0) and EMG activity after the elimination of the interferences (Corrected) in five subjects who received left sided unilateral interference

Figure 15 Timeline and the graphical analysis of the EMG activity at baseline (T0) 
and EMG activity after the elimination of the interferences (Corrected) in all subjects.................................................................................................. 33 


\section{CHAPTER 1. INTRODUCTION}

It is documented in adult oral health surveys that although the percentage of edentulous individuals has been steadily decreasing over the past years, the total number of edentulous subjects continues to rise as a result of increased life expectancy, increasing population, and elevated level of dental disease (Zarb, 1983). Therefore, one may expect that the functional problems associated with edentulism involving stability and retention (which results in diminished chewing efficiency, compromised speech, and decreased social interaction) may be more commonly seen in the portion of the population of greater age. Further, it is suggested that compromised dental function may lead to dietary inadequacies and systemic changes favoring illness (Bethesda, 1988; Finbarr, 2001; Piancino, 2005; Todd, 1991). These factors indicate that the problems limiting treatment of edentulism will not only affect the most fragile members of the dental profession's subject pool, but will also continue to challenge dentists, particularly as the ability to adapt to conventional complete dentures diminishes with age.

Not all functional problems associated with complete denture therapy can be attributed to the "lack of adaptability" of edentulous subjects. The design and fabrication of complete dentures can be wrought with errors and if left unaddressed may substantially lessen the quality of the resulting prosthesis (Keubker, 1984). In 1984, William Keubker published an article that outlined specific denture problems, the diagnostic procedures used to identify them, and the clinical treatment necessary for correction. While no clinician intends to introduce errors in complete denture therapy, it often happens, and can result in an extremely frustrating experience for both the subject and the clinician. In the case where errors are not avoided in fabrication, they may be lessened or eliminated during placement. Of particular interest is the effect that errors in occlusion have on the denture subjects, both psychologically and physically.

It seems intuitive that a more stable prosthesis should be more controllable to the wearer, and thus more effective. This characteristic and result was seemingly confirmed in a project by Garrett. He found that by correcting the occlusion and relining an unstable, poorly fitting prosthesis with a soft lining material the stability of the prosthesis and the activity and force of the masseter muscles was increased bilaterally (Garrett, 1996). In this same report, Garrett suggests that stability of a prosthesis may be gained from both the appropriate fit of the intaglio (tissue) surface and from accurate occlusal surface.

An Occlusal Interference is any tooth contact that inhibits the remaining occlusal surfaces from achieving stable and harmonious contacts (Glossary of Prosthodontic Terms, Lefebvre, 2005). Keubker identifies occlusal errors (a.k.a., occlusal interferences) as a possible cause of "generalized soreness" that may be associated either with the ridge of edentulous arch or the muscles of face and mastication (Keubker, 1984). What occurs in edentulous individuals wearing complete dentures that have interferences is the manipulation of the masticatory muscles to an accommodated (or habitual) position, a situation that is known to occur in dentate persons (McHorris, 1979). The specific effect 
that accommodation has on the masticatory musculature of edentulous subjects during rest or function is poorly defined in dental literature. While Keubker states, "certainly the best approach is to avoid as many problems as possible" (Keubker, 1984), the occurrence of this event in a busy dental practice is all too common. EMG studies have shown a positive correlation between muscle activity and chewing force (Hugger, 2008). In addition alterations in occlusal relation affect the EMG activity of muscle (Tallgren, 1995). In light of the increasing edentulous population (in both number and age), the systemic effects that poorly functioning dentures may have on their owner, and the overall decrease in adaptability of the aging edentulous population to complete dentures, the effect of errors must be more clearly identified. 


\section{CHAPTER 2. REVIEW OF LITERATURE}

\section{Studies on edentulous subjects}

Edentulous persons wearing conventional complete dentures are at an expected disadvantage (when considering masticatory performace) when compared to dentate individuals. In fact, the chewing efficiency of the denture wearer is less than one-sixth that of the subject with a natural dentition. Obviously, dentures act as poor functional replacements of natural dentitions (Kapur, 2006). Some of the factors to be considered in diminished chewing efficiency are the following:

(1) Ability to generate high forces with masticatory muscles,

(2) Ability to coordinate the masticatory musculature during function and rest, and

(3) Stability of the prosthetic dentition during function and rest.

While motor discrimination seems to be intact in denture wearers, their inability to tolerate high masticatory pressures (due to sensitivity of mucosa, lack of proprioception, etc) may prevent them from exerting discriminatory forces comparable to those applied by persons with natural dentitions (Kapur, 1984). In response to a decreased magnitude of occlusal-directed forces created by edentulous subjects, one can't help but wonder how the masticatory musculature may accommodate for decreased loading ability. Interestingly, it has been found that denture wearers with either superior or poor chewing ability exert similar biting forces during mastication. What's different between the two groups (superior versus poor chewing ability) is the ability to coordinate the masticatory musculature from the left side to the right. Left and right masseter muscle electromyographic (EMG) activity was recorded during a series of masticatory tests performed by Garrett in 1995. In a group of successful denture wearers, they found that the total mean EMG activity of the masseter muscles of the preferred and non-preferred (which side they preferred to chew on) side did not differ between the two groups significantly $(\mathrm{p}>0.05)$. In other words, successful denture wearers are able to coordinate the activity of the left and right masseter muscles during function (Garett, 1995). Masseter muscles are not the lone proprietors of the title of "the muscles of mastication."

\section{Studies on dentate subjects}

Bakke and Moller (1980) in their study concluded that maximum bilateral occlusal stability is a prerequisite for optimal neuromuscular generation of well adjusted bilateral clenching forces: optimal occlusal stability appears to facilitate bilateral central motor commands of equal strength. Unilateral occlusal instability appears to distort central motor commands to the paired jaw elevator muscles; the distortion seems to be the result of chaotic information from the periphery.

Ingervall, in 1982 conducted a project where the activity of the masseter and temporalis muscles was studied by EMG in a group of subjects. Twenty-four subjects 
were divided into two groups: those with unilateral balancing side interferences and those without interferences. The response of these muscles was then recorded. In both groups the EMG recordings were made during postural activity and various functions of the masticatory system. In the interference group, EMG recordings were repeated on two occasions following occlusal adjustment. The postural muscle activity was significantly lower in the interference than in the control group while there was no difference during maximal bite (Ingervall, 1982).

Christensen and Rassouli (1995) performed a study on twelve subjects by placing a rigid unilateral intercuspal interference (mean height of .24 mm) and recording EMG activities of right and left masticatory muscles during brisk and forceful clenching. They noted that both masseter and the temporalis muscles show functional heterogeneity. Frequently there was a motor facilitation of the masseter on the side of interference and a significant motor inhibition on the opposite side.

Riise and Sheikholeslam (1982) performed their study on eleven subjects with a $0.5 \mathrm{~mm}$ occlusal interference and studied its effect on the pattern of activity of the anterior temporalis and the masseter muscles. They found that one hour after the insertion of an occlusal interference, there were no significant changes in the pattern of the postural activity except in two subjects who showed increased activity in temporalis muscle on one side. They concluded that the temporalis muscle is among the first muscles to be affected by anxiety and stress and could develop hyperactivity. They explained the asymmetrical hyperactivity in either the right or left temporal muscle was an attempt to shift the mandible to avoid the interference.

Michelotti, et al. (2005) in his research study concluded that the introduction of active occlusal interference in healthy individuals alters the pattern of habitual activity of the masseter muscles. However, contrary to the "hyperactivity" hypothesis, the muscle activity dropped following the application of the interference. The possible cause of which was an avoidance behavior developed in response to occlusal discomfort.

Li, Jiang, et al. (2007) conducted a study on six healthy individuals by placing a $.5 \mathrm{~mm}$ occlusal interference and measured the EMG activity on the masseter and the temporalis muscles bilaterally. On the third day of placement of interference there was increased postural activity of the temporalis muscle on the side of the interference. On the third day and the sixth day with the interference the EMG activity of the tested muscles during maximal voluntary contraction was significantly reduced. They analyzed their results on the basis that the jaw muscles must contribute to the stabilization and hence reduce the magnitude of maximum contraction to avoid the damage to the structure involved in the compensatory stability.

Finally, the stability of one's dentition, whether natural or prosthetic may play a role in masticatory performance. When restoring an edentulous mouth using a conventional complete denture, the ultimate goal is to produce a prosthesis that is as stable and retentive as possible. Undoubtedly, differences exist between naturally dentate subjects, and those restored with conventional complete dentures on a multitude of 
abilities and characteristics. This project was carried out to establish the effect that a deviation from the "ideal" (in the fabrication and delivery procedures of conventional complete dentures) may have on the musculature (EMG activity) of an edentulous subject and also to examine how the presence and absence of these inaccuracies influence the subject's perceived satisfaction with their prostheses.

\section{Electromyography}

Masticatory function may be analyzed using both objective and subjective methods. Electromyographic activity evaluation is an objective method that provides the masticatory muscle behavior in many situations during the function. The technique is based on capturing electric potentials from the muscles in action during natural and voluntary movements (Pruzansky, 1952). However; there are few eletromyographic (EMG) studies on the effects of tooth loss and complete denture use (Tallgren, 1995). Considering the evidences about the masticatory muscle behavior, electromyography evaluations seem to be an important auxiliary instrument employed in the clinical diagnosis of the stomatognathc system function (Moyers, 1949).

Electromyography (EMG) is a technique for evaluating and recording the activation signal of muscles. The Instrument used to measure the muscle activity is the electromyograph. The record obtained is called the electromyogram.

An electromyograph detects the electric potential generated by muscle cells when these cells contract, and also when the cells relax. The motor response (muscle) may be characterized by its amplitude, duration, and wave form. The amplitude is measured from the baseline to the top of the peak of the motor response and is expressed in millivolts or microvolts

When processing EMG data there are three commonly applied steps:

(1) Rectifying the raw data (original waveform): This is a process of changing the signal from a bi-polar signal (with both positive and negative phases) into a unipolar signal (with only positive phases) see Figure 1. However, this stage of the processing is not usually displayed on the screen. NOTE: Rectification can also change everything into negative phases, but that is less commonly done.

(2) After the signal is rectified an "Averaging Filter" is usually applied. This produces what the BioPAK program labels as the "AVERAGED EMG" window. This is a process that smoothes out the sharp up and down transitions of the original waveform and creates something that looks more like a plot of contraction strength see Figure 2. The filter in BioPAK is digital, but whether it is digital or analog, it includes a "time constant" that indicates how much the waveform is smoothed. Typically values between about 40 milliseconds and 100 milliseconds are used, with 100 creating a smoother result. The big advantage of 


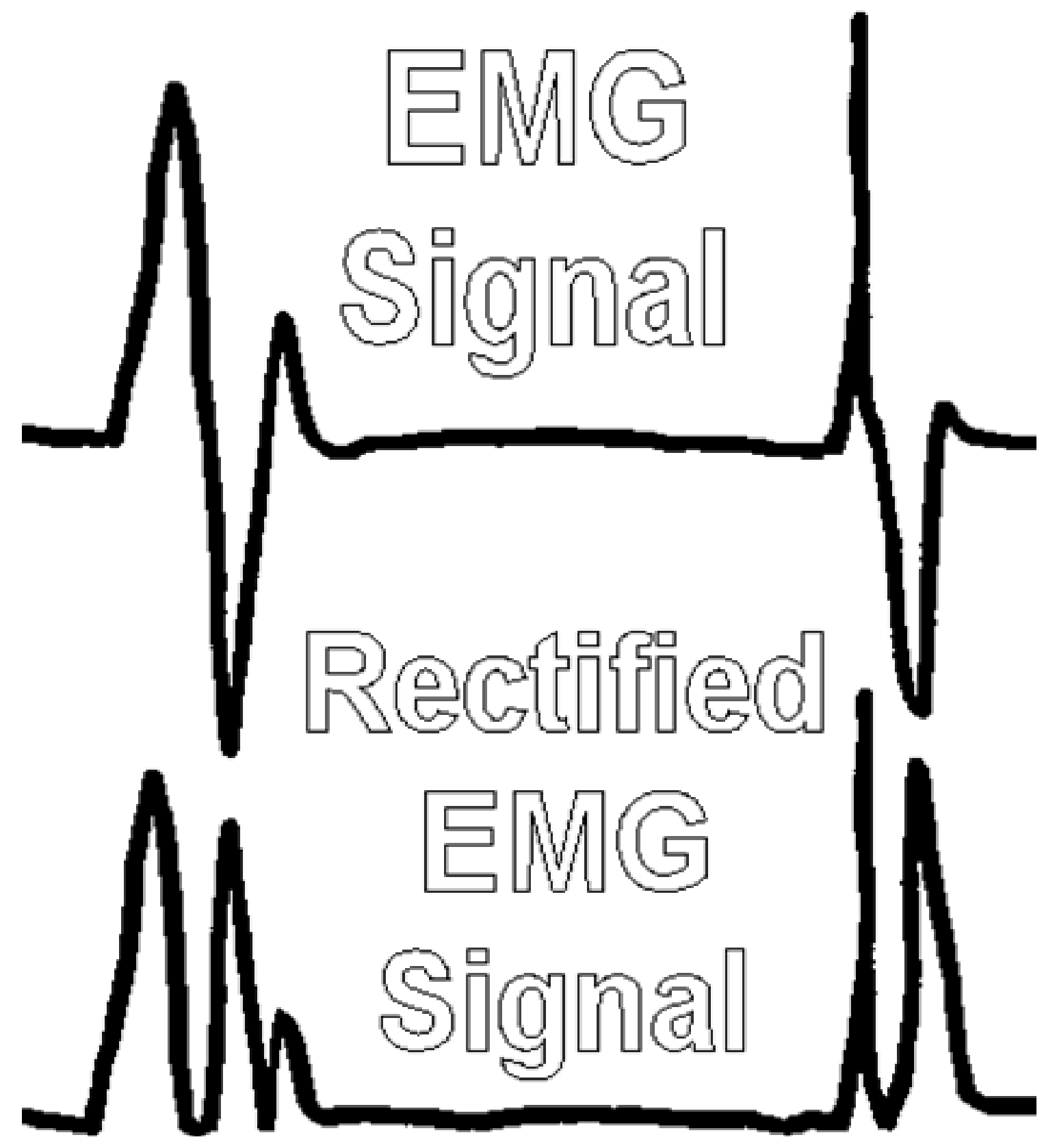

Figure 1 Rectification of raw EMG data 


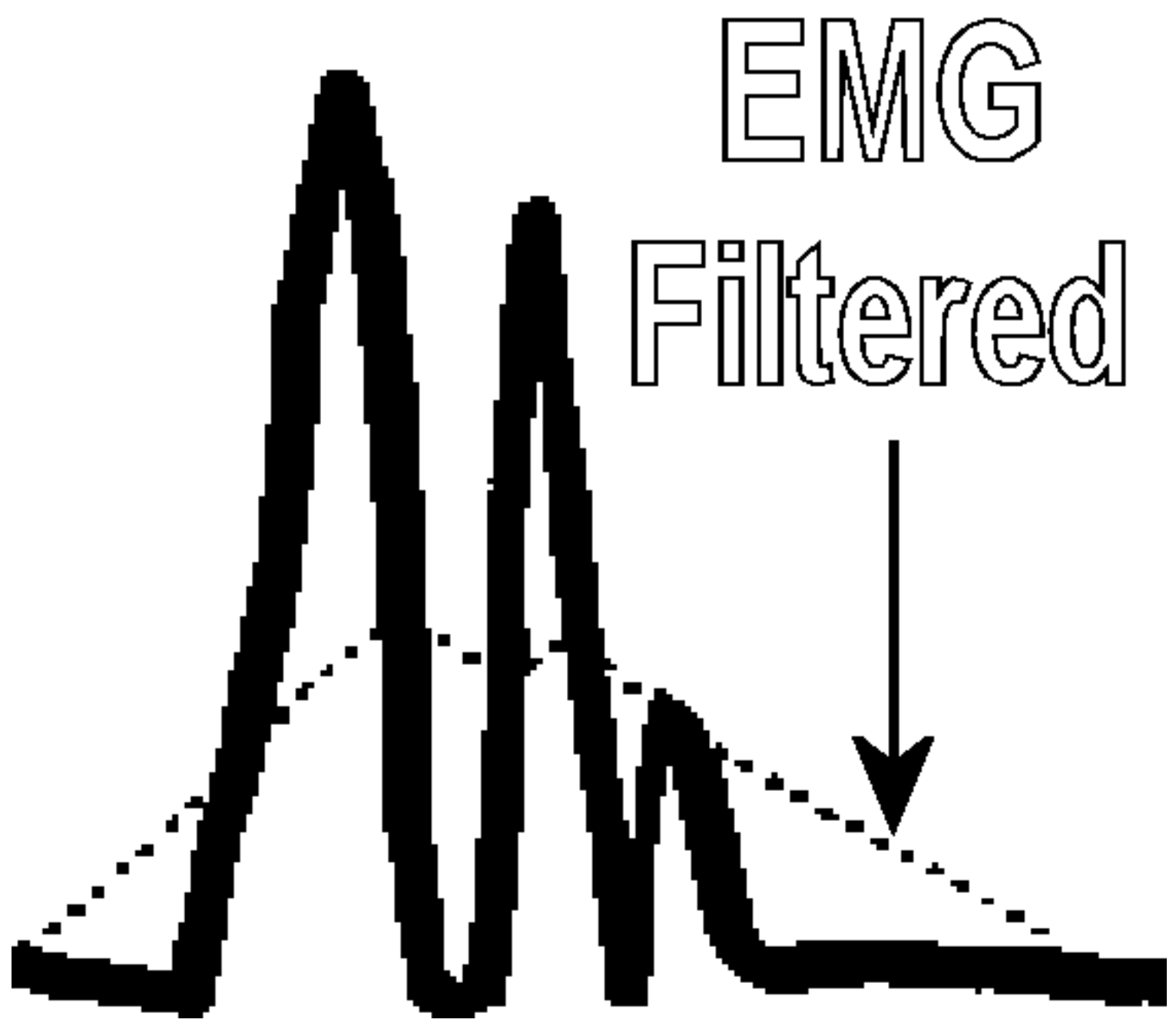

Figure 2 Application of averaging filter to the rectified EMG data 
digital filtering is that it does not add any time delay to the signal.

(3) When an area is "MARKED" by the operator, the program adds up all of the rectified values (all positive) within the current cursor window and then divides by the number of points summed, see Figure 3. This produces the EMG summary values in the upper right hand corner of the screen.

The average level is an arithmetic mean for each channel recorded, but only for the portion that lies within the cursor window, see Figure 4. For that reason when "MARKING" a trace, ideally you should adjust the cursor window width to precisely match the duration of the event you are marking. If that is not possible, then the window should be a bit narrower rather than wider and exclude the last part of the event. The effect of having the cursor window much wider than the event simply dilutes any difference that may exist between channels. This occurs because the areas that have no activity will look the same or at least very similar for all channels. If you mark two or more events, the program "averages the averages" for each channel. It just means your average is being calculated over a greater period of time and multiple events. The presentation of the EMG data on the computer screen is demonstrated in Figure 5.

The EMG recording has the following applications (Hugger, 2008):

(1) For analyzing neuromuscular function.

(2) To assess the neuromuscular chewing performance after prosthetic reconstructions.

(3) For recording influence of pain on neuromuscular system.

(4) For documenting effects of interventions on Temporomandibular disorders.

\section{T Scan}

The T Scan II uses the proprietary force sensor technology and software to quantify the occlusal contact data. The technique involves placing a sensor in the subject's mouth and asking the subject to close in maximum intercuspation (MIP) while the computer records a series of frames of the subject's tooth contacts. The sequential frames are collected at a sampling rate of $50 \mathrm{~Hz}$ and assembled into a movie that can be replayed. Occlusal contacts are represented as topographical images that describe the shape of the contact area, relative force, and surface area. Differences in occlusal force are shown by color ranging from red as the greatest force to blue as the least force, using the standard order of colors of the spectrum. These contour images of the tooth contacts provide an instant view of the areas of greatest tooth contact and relative force. The images are easily analyzed by the software by summing the force weighted surface area for comparison. These images will be used for correcting the occlusal error in the dentures and establishing a bilateral lingualised balanced occlusion (Olivieri, 1988). The presentation of the T Scan data on the computer screen is demonstrated in Figure 6. There have been several studies in the literature proving the validity of T Scan for quantifying occlusal data in dentate individuals (Makofsky, 2000; Maness, 1987; 


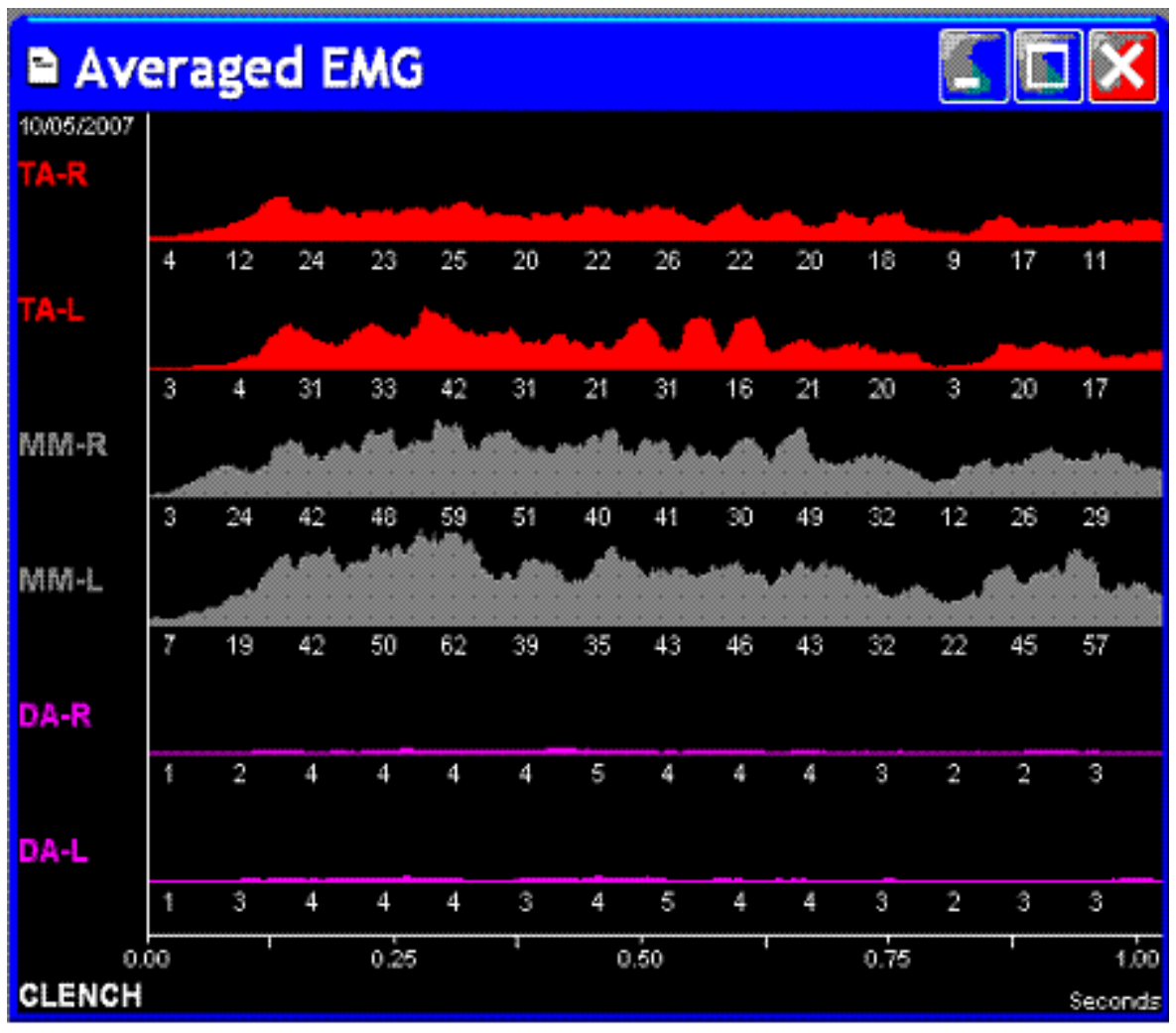

Figure 3 Averaged EMG data 


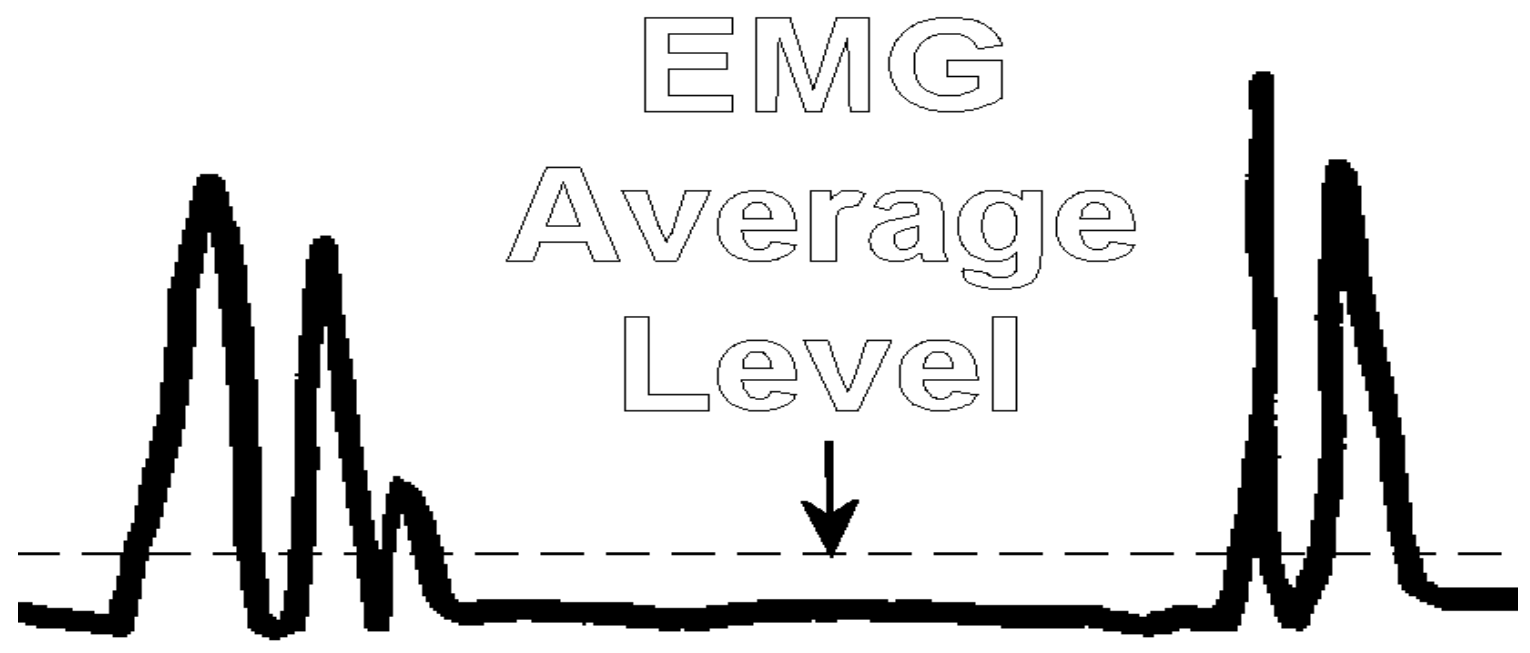

Figure 4 EMG average level

10 


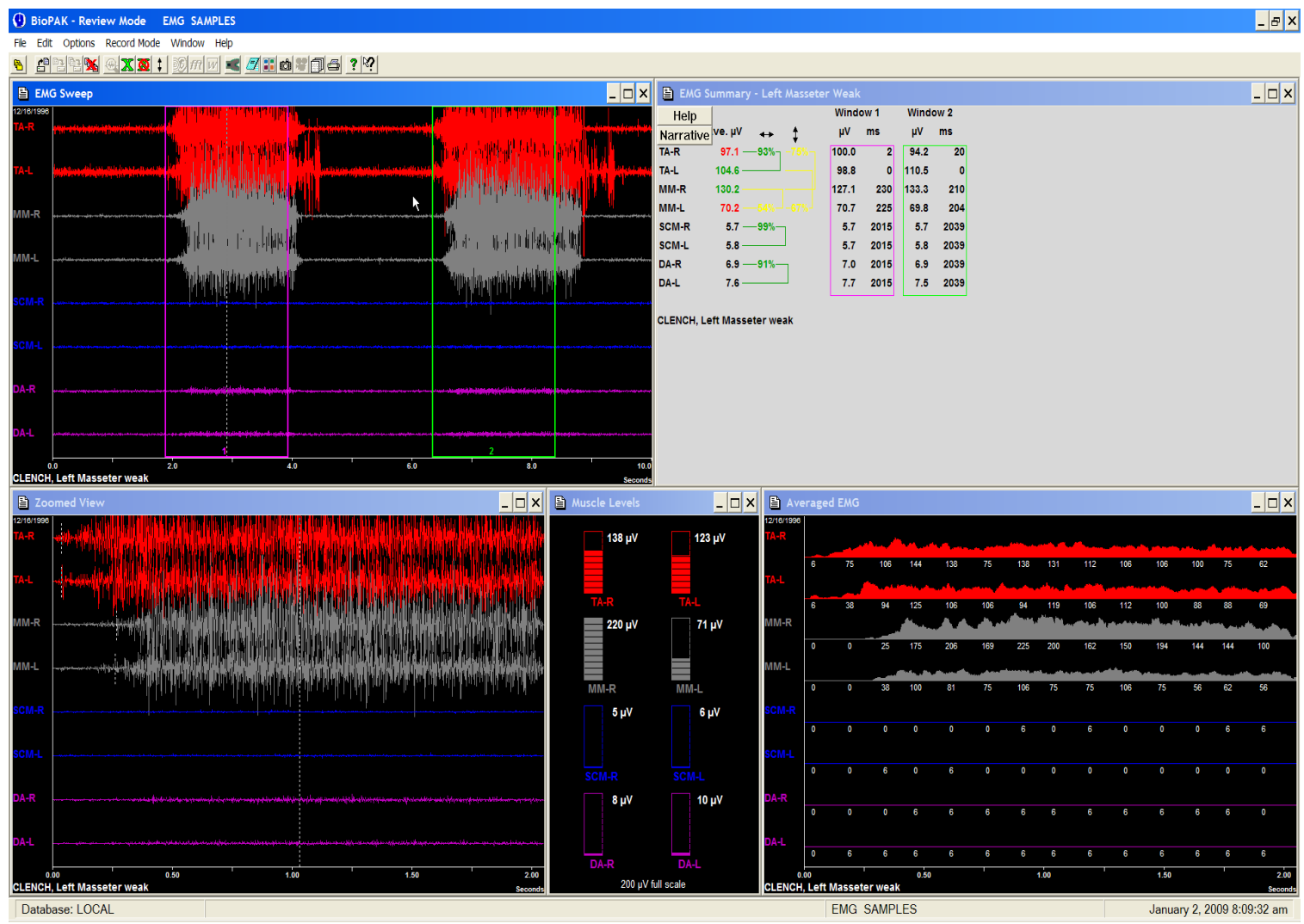

Figure 5 Presentation of EMG data on the computer screen 


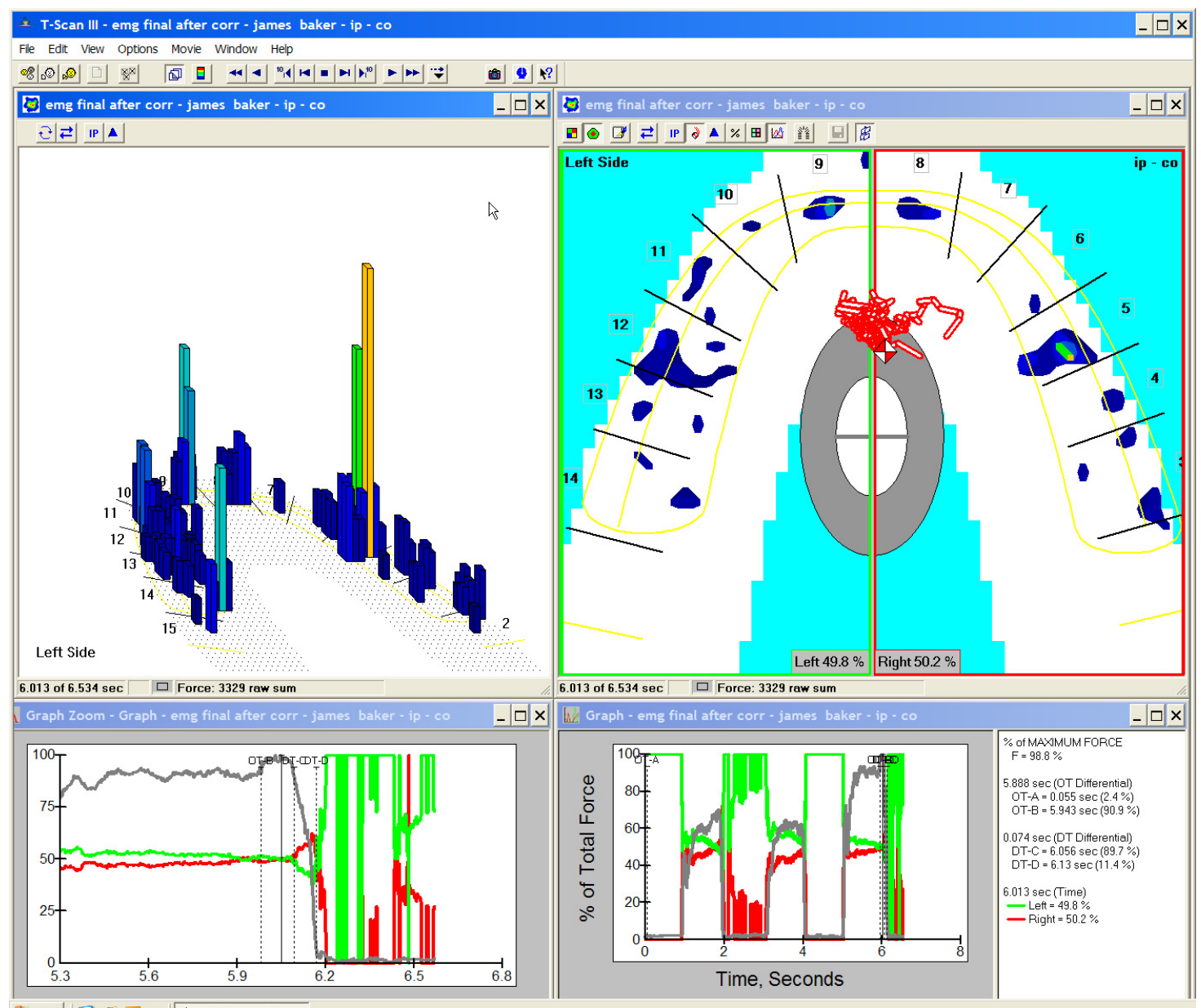

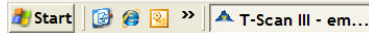

Figure 6 Presentation of $T$ Scan data on the computer screen 
Kerstein, 2001). In addition there are studies validating T Scan as a tool for diagnosing occlusal errors in complete denture wearers (Boening, 1992; Olivieri, 1998). 


\section{CHAPTER 3. RESEARCH OBJECTIVES}

Achievement of subjects satisfaction is one of the highest goals in the treatment of all dental subjects and more so the edentulous subjects. Dentists have thus tried to improve the subject's adaptation, chewing function, speech retention and stability by various approaches.

Movements of the mandible are influenced by proprioceptors in muscles, joints and mucosa. In the elderly there may be a delay in the central processing of nerve impulses. Studies have shown that the number of functional motor units, fast muscle fibers and the cross sectional area of the masseter and medial pterygoid muscles can decrease with age. Muscle tone can decrease by as much as $20 \%$ to $25 \%$ in old age, which probably explains the shorter chewing strokes and prolonged chewing time (Garrett, 1996). Thus if muscle tone and activity are already compromised we want to know and eliminate all the factors that would contribute to its decline. As such, specific aims and hypotheses for this study are:

\section{Specific aim I}

To monitor subjective complete denture subject response upon introduction and elimination of unilateral and bilateral occlusal interferences. Related hypothesis is complete denture subjects perceive the introduction and elimination of the occlusal interferences.

\section{Specific aim II}

To monitor CD subject muscle activity upon introduction and elimination of unilateral and bilateral occlusal interferences. Related hypothesis there is a reduction in muscle activity upon introduction of occlusal interferences and a return to baseline muscle activity upon elimination of the occlusal interferences. 


\section{CHAPTER 4. METHODS AND MATERIALS}

Ten subjects were chosen for this study. Subjects were screened and selected by Prosthodontic residents. Upon selection, all subjects were required to sign a form consenting to treatment and participation in the study. Each subject served as his or her own control to determine the effects of occlusion on muscle activity.

\section{Subject recruitment, screening and consent}

Subjects selected for inclusion in this study were qualified on the basis of a comprehensive evaluation. A complete medical history, dental history, and personal interview were conducted by the investigators to ascertain the current state of health and to identify any contraindications to participation in the study. Subjects of either gender who met the selection criteria were sequentially enrolled into the study. The physical conditions of all subjects were medically and dentally appropriate to undergo all planned procedures. Indications and contraindications for participation in the study were weighed against the health status of potential participants and possible alternative dental restorative options.

After obtaining the institutional review board approval for the study, subjects were recruited by solicitation through advertisements, review of dental records currently on file at the UTHSC, Memphis Dental School, and following identification in the normal course of subject management at the UTHSC, Memphis Dental School. The experimental population consisted of ten subjects. To control extraneous variables a properly calibrated EMG activity recorder was used. The Biopak EMG which is ADA approved and the T Scan II which is the improved version of the T Scan I were used in this study.

Solicitation through advertisements included notices on bulletin boards, newsletter solicitations, electronic mail solicitations, and both written and verbal announcements to individual practitioners. Advertisements were neither misleading nor coercive to potential subjects and made no claims, either explicitly or implicitly, that the proposed treatment is safe and effective or equivalent or superior to other treatment alternatives. Advertisements included (1) names and addresses of investigators, (2) the purpose of the research and eligibility criteria used to admit subjects, (3) a description of the benefits of the treatment, (4) the location where treatment will be provided, and (5) the name of a person to contact for further information regarding the research project and a direct phone number.

Recruitment of subjects into the research project was accomplished by reviewing treatment records currently on file in the UTHSC, Memphis Dental School. Evidence of the subject's signature on a standard release form within the treatment record was present prior to including the subject in the project. The subject's primary dental care provider was included in all communications with the subject. Letters sent to recruit these subjects 
were co-signed by the subject's primary dental care provider.

Health questionnaires commonly use in the dental faculty practice clinic at the University of Tennessee Health Science Center, School of Dentistry, were completed by all subjects prior to the screening examination. All subjects were screened by investigators, who determined if each subject satisfied participation criteria. Initial subject interviews included the following:

(1) Discussion of the research project,

(2) Review of medical and dental histories,

(3) Measurement of blood pressure and pulse, and

(4) Standard intraoral and extraoral dental examinations.

Following the initial evaluation, and in the event that a subject fulfilled inclusion criteria, a panoramic radiograph was made and evaluated. Appropriate periapical radiographs were made, if additional radiographic information is deemed necessary. These dental radiographic exposures are usual and customary for screening of subjects with dental problems. A file of information on each subject screened was be maintained in the advanced Prosthodontic dental clinic.

Once accepted into the study population, each subject signed a consent form designed specifically for this study and approved by the UTHSC, Memphis Institutional Review Board. Treatment did not begin without legally effective informed consent signed by the subject, or the subject's legally authorized representative. The consent process involved the following:

(1) Explaining to subjects about the research project and associated dental treatment,

(2) Ensuring that subjects understood this information,

(3) Reviewing the subjects financial responsibilities,

(4) Permitting subjects adequate opportunities to consider all treatment options,

(5) Responding to subject questions, and

(6) Obtaining the subject's voluntary consents to treatment.

Signed consent forms were securely kept on record in the department of Prosthodontics at the UTHSC, Memphis. Participation in this study presented minimal risk to subjects. These risks were described in detail in the consent form and carefully reviewed with each subject. Alternative treatment options for these subjects include Implant supported overdentures, Implant supported fixed complete denture, Hybrid appliance, and no treatment.

The proposed dental treatment was provided by prosthodontic residents who are training to specialize in Prosthodontics. In the event that dental problems arise, the investigators would treat the adverse effects accordingly. Each subject benefitted greatly from the prosthetic rehabilitation provided by the study and received the new dentures free of cost. The fee for similar treatment at a private clinic is approximately $\$ 3000$. The total financial responsibility of each subject reflected the cost of other dental procedures 
needed to restore and maintain the health of the oral cavity. Subjects were required to pay appropriate professional fees for these services at the time the services are rendered, as is customary in the UTHSC, Memphis.

\section{Inclusion and exclusion criteria}

Subjects were enrolled in the study if they met the following inclusion criteria:

(1) Subjects had to be present for treatment and follow-up examination according to the scheduled requirements of the research project. Subjects had to be in the age range of 18-75 years. Subjects were selected regardless of sex, race or ethnicity.

(2) Subjects had to be free of uncontrollable diabetes, existing malignancy, and not be receiving immune suppressive therapy, such as radiation therapy, chemotherapy, or chronic steroid therapy. Subjects with advanced cardiovascular disease, pulmonary disease, renal disease, liver disease, or significant alcohol ingestion were excluded from the study. Subjects who were pregnant were excluded from the study.

(3) Subjects who had root pieces, very deep undercuts, bony exostosis or any oral pathology were excluded from the study.

(4) They had to be free of any Temporomandibular joint, musculoskeletal or neurological disorders.

Following active treatment, subjects were asked to report to the advanced Prosthodontic Dental Clinic at the University of Tennessee Health Science Center for follow-up care and evaluation. Subjects who were not available to appear for follow-up care and evaluation were not accepted into this study. Diagnostic casts were made and, when necessary, mounted in a semi-adjustable articulator.

\section{Characteristics of the subject population}

The sample size consisted of ten subjects. They were randomly divided in to two groups. Group A received Unilateral right interference and Group B received left unilateral interference. All the subjects were classified by the Prosthodontic Diagnostic index (PDI). Three of the subjects were class I, two were Class II, two were class III and the remaining three were Class IV. The subjects were in the age group of 45-75yrs. Seven subjects were female and three were male. Seven subjects were white and the remaining three were African American.

\section{Study design}

This was a prospective, non-blinded, randomized, controlled clinical study. Human subjects who met the inclusion criteria were sequentially enrolled for treatment. All enrolled subjects received one set of complete dentures. 


\section{Typical research procedures}

Routine diagnostic procedures

After agreeing to participate in the study and providing legal consent, subjects were seen by a prosthodontic resident for diagnostic evaluation. A thorough oral examination was performed to:

(1) Assess health status.

(2) Identify oral pathologies that either require treatment or exclude the subject from the study.

(3) Analyze available bone and mucosa in the edentulous arches.

Other medical tests and/or consultations were acquired when indicated. Comprehensive hard and soft tissue examinations was performed to rule out undiagnosed malignancies, dysplastic oral and head and neck lesions, or zones of inadequate keratinized gingiva. Each subject was questioned about their history of parafunctional habits. The Temporomandibular joints were evaluated for deviations in function, joint sounds and pain.

Once a subject was diagnosed medically, dentally, psychologically, functionally, and anatomically to be a good candidate for complete denture therapy, and was committed to pursuing treatment as a participant in this study, a complete diagnostic work-up was accomplished. A panoramic radiograph was made in order to verify that no contraindicated abnormalities are present (i.e., root tips, cysts, anatomic anomalies, etc.) and to identify the locations of the mental foramina.

Intraoral and extraoral photographs were made of each subject to demonstrate clinical conditions present at the beginning, middle, and end of the study. The views to be included were the following:

(1) Extraoral, smile

(2) Extraoral, repose

(3) Intraoral, right side, maximum intercuspation

(4) Intraoral, right side, right working

(5) Intraoral, right side, right non-working

(6) Intraoral, left side, maximum intercuspation

(7) Intraoral, left side, left working

(8) Intraoral, left side, left non-working

(9) Intraoral, front, maximum intercuspation

(10) Intraoral, front, protrusive

Additional photographs were made, as needed to demonstrate clinical procedure. 


\section{Routine prosthodontic procedures}

Alginate impressions were made of the maxilla and mandible to produce diagnostic casts that were used to fabricate custom impression trays. Master impressions were made utilizing the border molded, custom trays and polysulfide impression material. The master casts was then mounted on a semi-adjustable dental articulator using custom fabricated bases and wax rims. Position of the denture teeth was driven primarily by esthetic placement of the maxillary anterior teeth. Mandibular posterior teeth were set over the crest of the ridge (at half the height of the retromolar pad area of each side) utilizing a curved guide plane. The maxillary posterior teeth (Ortholingual type, Ivoclar Vivadent, Intl.) were set with the lingual cusps contacting the central fossae of the opposing mandibular molars and premolars in a lingualized, bilaterally balanced occlusal scheme. The completed diagnostic setup was verified intraorally and with a laboratory remount at the wax try in appointment. Any necessary adjustments were made at that point. The dentures were processed in a conventional manner. On the day of delivery any needed adjustments to the intaglio, cameo, and occlusal surfaces of the complete denture were performed. A clinical remount was accomplished, and any existing interferences (likely caused by processing error) were removed.

\section{Atypical research procedures}

The timeline of the project is illustrated in Figure 7. T Scan was used to verify that an accurate occlusal adjustment had been accomplished. Once done, EMG was recorded to assess masticatory muscle activity. All the ten dentures were returned to the remount indices, and composite resin was used to place bilateral artificial posterior interference on the occlusal surface of the most posterior teeth of quadrant III and quadrant IV of the mandibular complete denture. This resulted in an opening of the subject's occlusal vertical dimension by $2 \mathrm{~mm}$ (recorded by the position of the anterior guide pin of the articulator). Subjects were given thirty minutes to adapt to the interference and then T Scan was used to record the presence, and verify the position and timing of the artificially placed interferences. Bilateral contact of the interferences occurred simultaneously and with the same degree of force. Using a questionnaire (Appendix A) specially formulated for this project, subjects reported their level of perception of the inaccuracy of the bite and then an EMG recording was made.

Five complete dentures were randomly selected and returned to the remount indices the bilateral interferences were removed and composite resin was used to place an artificial posterior interference on the occlusal surface of the most posterior tooth of quadrant III of the mandibular complete denture. This resulted in an opening of the subject's occlusal vertical dimension by $2 \mathrm{~mm}$ (recorded by the position of the anterior guide pin of the articulator). Subjects were given thirty minutes to adapt to the interference and T Scan was used to record the presence, and verify the position and timing of the artificially placed interferences. Using a questionnaire (Appendix A) specially formulated for this project, subjects reported their level of perception of the 


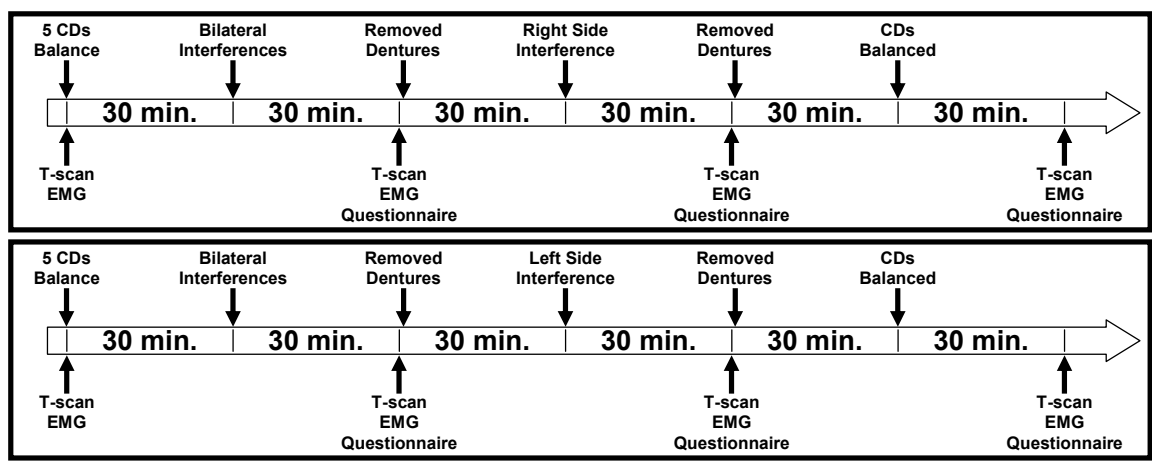

\section{Figure 7 Timeline of the project}


inaccuracy of their bite and then an EMG recording was made. The remaining five complete dentures were returned to the remount indices the bilateral interferences were removed, and composite resin was used to place artificial posterior interference on the occlusal surface of the most posterior tooth of quadrant IV of the mandibular complete denture. This resulted in an opening of the subject's occlusal vertical dimension by two millimetres (recorded by the position of the anterior guide pin of the articulator). Subjects were given thirty minutes to adapt to the interference and then T Scan was used to record the presence and verify the position and timing of the artificially placed interferences. Using a questionnaire (Appendix A) specially formulated for this project, subjects reported their level of perception of the inaccuracy of the current prostheses and then an EMG recording was made. All complete dentures were returned to the remount indices where the artificial occlusal interferences were removed to once more reach an "accurate occlusion". This "accurate occlusion" was verified by T Scan. Once verified, EMG signal levels of the masticatory muscles bilaterally were recorded. Subjects were asked to fill out a questionnaire (Appendix B) and were released from the clinic. During each recording, each subject was seated comfortably and relaxed in a dental chair. The subjects were asked to fix a target on the wall, $90 \mathrm{~cm}$ away, to avoid lateral movements of the head. They were then asked to clench maximally.EMG for this task was recorded for the anterior temporalis and master muscles (bilaterally) by placing self-adhesive, disposable, silver-chloride, bipolar electrodes over the anterior portion of the bulge of each muscle while the subject was asked to clench maximally. To reduce the electrode impedance, the skin was cleansed with $91 \%$ isopropyl alcohol that was allowed to evaporate before placing the electrodes. The electrodes were not removed in between the recordings thus maintaining the same positions of the electrodes in different recordings. For each recording, every single subject was asked to clench their teeth maximally and EMG measurements were recorded.

\section{Surface EMG recordings}

Surface EMG signals were recorded with the Biopak EMG. Two electrodes (Duotrode silver/silver chloride EMG electrodes) were located on the greatest bulge of the anterior portion of the masseter and temporalis muscles bilaterally, with an interelectrode distance of $20 \mathrm{~mm}$. This electrode arrangement and placement provided small sensitivity to electrode displacements and good repeatability of EMG variables. Before electrode placement, the skin was lightly cleaned with alcohol.

\section{Follow-up examination and data management}

All clinical and research data was entered into a database management system on a personal computer for rapid recall and collection. Statistical analysis of the data was performed by the investigators. Each subject was examined and experimental data collected by the investigators utilizing the BioPak EMG. 


\section{CHAPTER 5. RESULTS}

The data collected for each variable was summarized using descriptive statistics means, standard deviations, and students paired T-test. Students paired T-test was applied to test the null hypothesis that there was no statistical difference among the results obtained at the four measurement occasions. The significance level was set at $\mathrm{P}<0.05$. The results of the questionnaires were inconsistent and uncorrelated with clinical occlusal conditions throughout the study.

The EMG activities of Group A, subjects who were planned to receive unilateral right interference and Group B, subjects who were planned to receive unilateral left interference are listed in the Table 1 and Table 2, respectively. In the single subject, all EMG data were the arithmetic means of the four surface EMG recordings. Student's Ttest was used to analyze the data.

(1) Comparison of baseline EMG and EMG with bilateral interferences:

- When the EMG activity at baseline was compared with the EMG activity after the introduction of the bilateral interferences in five subjects who would recieve right sided unilateral interference at significance level of .05 no significant difference was found between the two, see Figure 8.

- When the EMG activity at baseline was compared with the EMG activity after the introduction of the bilateral interferences in 5 subjects who would recieve left sided unilateral interference at significance level of .05 no significant difference was found between the two, see Figure 9.

- When the EMG activity at baseline was compared with the EMG activity after the introduction of the bilateral interferences in all subjects at significance level of .05 no significant difference was found between the two, see Figure 10. The analysis of questionnaire did not reveal any significant findings. The subjects did not perceive the inaccuracy in their bite.

(2) Comparison of baseline EMG and the EMG with unilateral interferences:

- When the EMG activity at baseline was compared with the EMG activity after the introduction of the unilateral interferences in 5 subjects who received right sided unilateral interference at significance level of .05 no significant difference was found between the two, see Figure 11.

- When the EMG activity at baseline was compared with the EMG activity after the introduction of the unilateral interferences in 5 subjects who received left sided unilateral interference at significance level of .05 there was a significant reduction in the EMG activity of the left masseter as compared to the baseline, see Figure 12.The analysis of questionnaire did not reveal any significant finding. 
Table 1 EMG value in five subjects at baseline (Base EMG), introduction of bilateral interferences (Bilateral interference), introduction of right unilateral interference (Unilateral interference) and elimination of interference (EMG corrected)

\begin{tabular}{|c|c|c|c|c|c|c|c|c|c|c|c|c|c|c|c|c|}
\hline \multicolumn{2}{|c|}{ Subject \# } & \multicolumn{3}{|c|}{ Base EMG } & \multicolumn{4}{|c|}{ Bilateral interference } & \multicolumn{4}{|c|}{ Unilateral interferences } & \multicolumn{4}{|c|}{ EMG corrected } \\
\hline & Mass & Mass & Tem & Tem & Mass & Mass & Tem & Tem & Mass & Mass & Tem & Tem & Mass & Mass & Tem & Tem \\
\hline & Right & Left & Right & Left & Right & Left & Right & Left & Right & Left & Right & Left & Right & Left & Right & Left \\
\hline 1 & 28.9 & 29.2 & 12.9 & 24.8 & 21.3 & 23.3 & 17.2 & 31.1 & $\begin{array}{l}\text { INT } \\
12.1\end{array}$ & 22 & $\begin{array}{l}\text { INT } \\
19.6\end{array}$ & 40.6 & 20.7 & 17.4 & 15.2 & 9.9 \\
\hline 2 & 63.6 & 43.9 & 56.4 & 51.3 & 69.1 & 23.1 & 47.9 & 23.3 & $\begin{array}{l}\text { INT } \\
70.6\end{array}$ & 22.6 & $\begin{array}{l}\text { INT } \\
56.6\end{array}$ & 27.8 & 68.5 & 25.7 & 43.9 & 30 \\
\hline 3 & 21.3 & 37.1 & 23.8 & 41.8 & 31.7 & 37.4 & 17.4 & 18.2 & $\begin{array}{l}\text { INT } \\
14.9\end{array}$ & 17.5 & $\begin{array}{c}\text { INT } \\
12.21\end{array}$ & 42.9 & 18.9 & 43.1 & 29.5 & 34 \\
\hline 4 & 30.3 & 25.4 & 65.9 & 33.6 & 30.2 & 26.4 & 45.7 & 28.7 & $\begin{array}{l}\text { INT } \\
28.6\end{array}$ & 27.7 & $\begin{array}{l}\text { INT } \\
29.7\end{array}$ & 46.1 & 19.2 & 26.3 & 30.6 & 45.5 \\
\hline 5 & 41.6 & 42 & 16.5 & 22.3 & 45.6 & 58.2 & 24.7 & 30 & $\begin{array}{l}\text { INT } \\
31.7\end{array}$ & 56.6 & $\begin{array}{l}\text { INT } \\
26.3\end{array}$ & 42.2 & 41.3 & 73.7 & 21.7 & 35.7 \\
\hline
\end{tabular}


Table 2 EMG value in five subjects at baseline (Base EMG), introduction of bilateral interferences (Bilateral interference), introduction of left unilateral interference (Unilateral interference) and elimination of interference (EMG corrected)

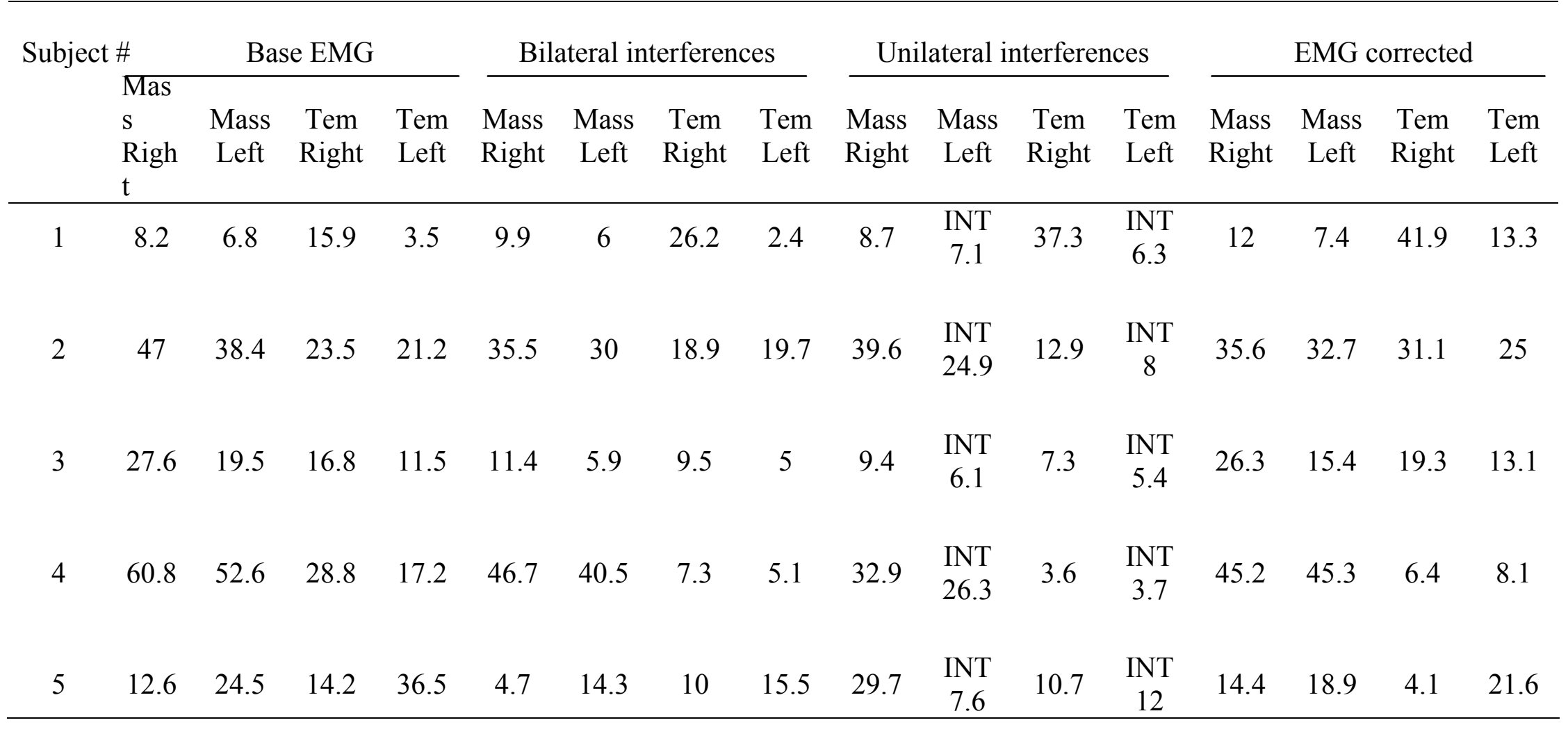



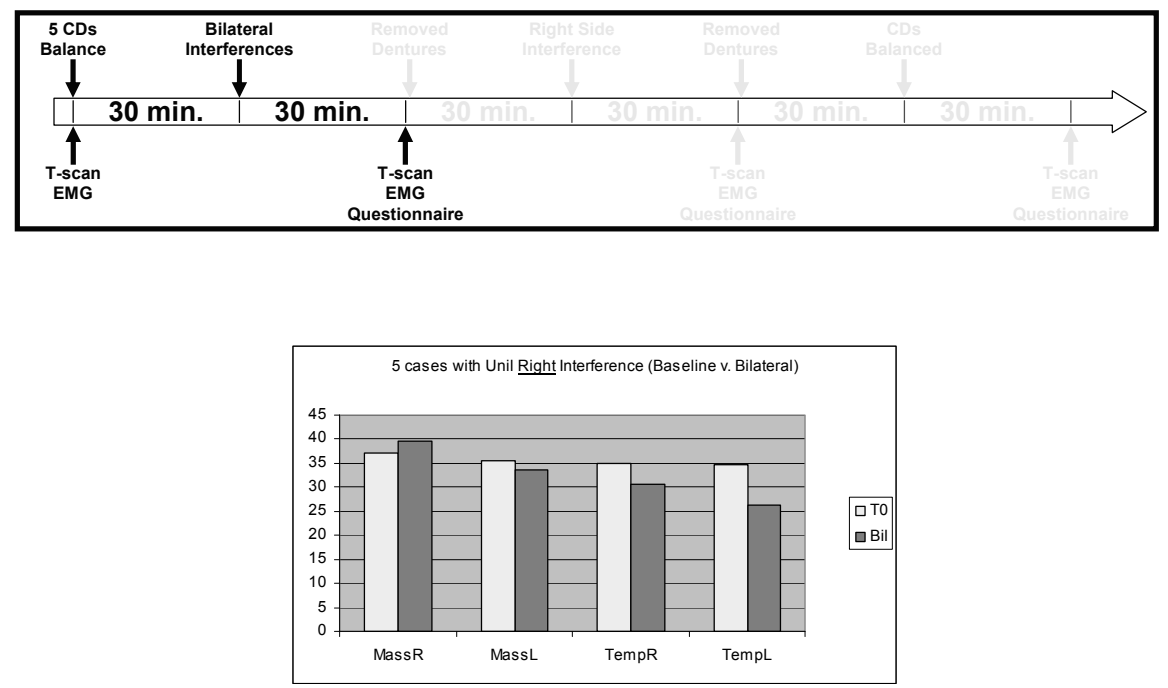

Figure 8 Timeline and the graphical analysis of the EMG activity at baseline (To) and EMG activity after the introduction of the bilateral interferences (Bil) in five subjects who would receive right sided unilateral interference

(Mass R-Right Masseter, Mass L-Left Masseter, Temp R-Right anterior Temporalis, Temp L-Left anterior Temporalis)

Note: At significance level of .05 no significant difference was found between the two. 

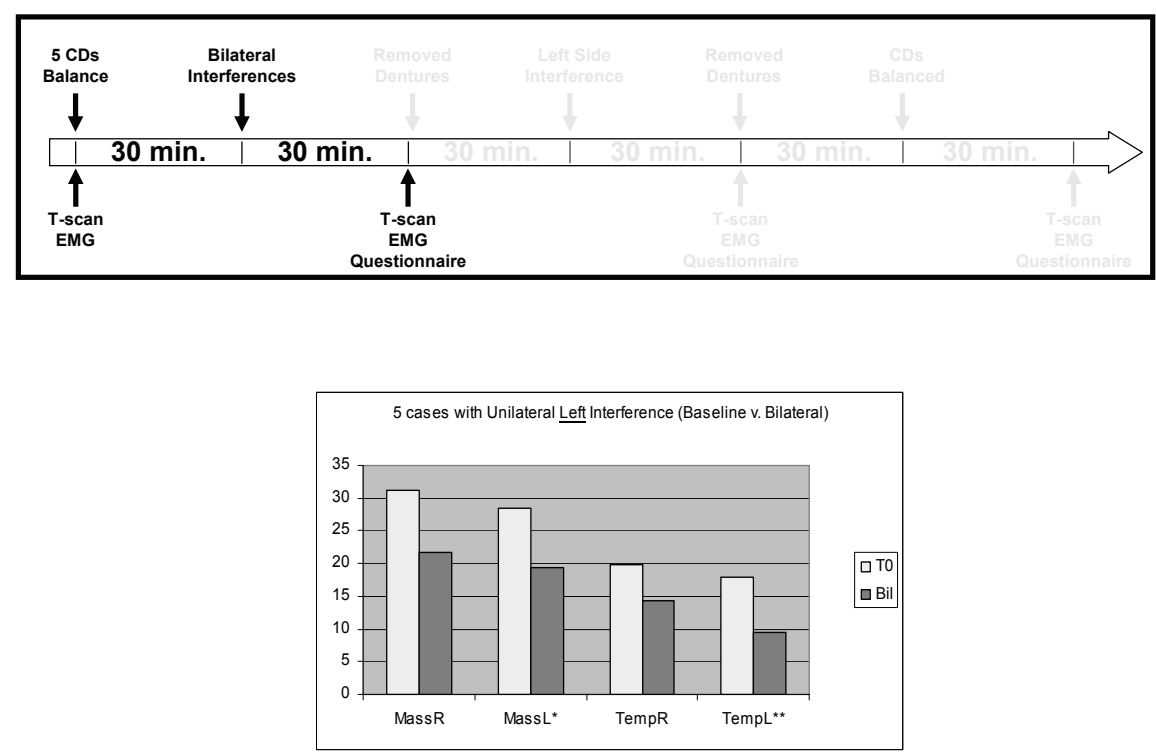

Figure 9 Timeline and the graphical analysis of the EMG activity at baseline (T0) and EMG activity after the introduction of the bilateral interferences (Bil) in five subjects who would receive left sided unilateral interference

(Mass R-Right Masseter, Mass L-Left Masseter, Temp R-Right anterior Temporalis, Temp L-Left anterior Temporalis)

Note: At significance level of .05 no significant difference was found between the two. 

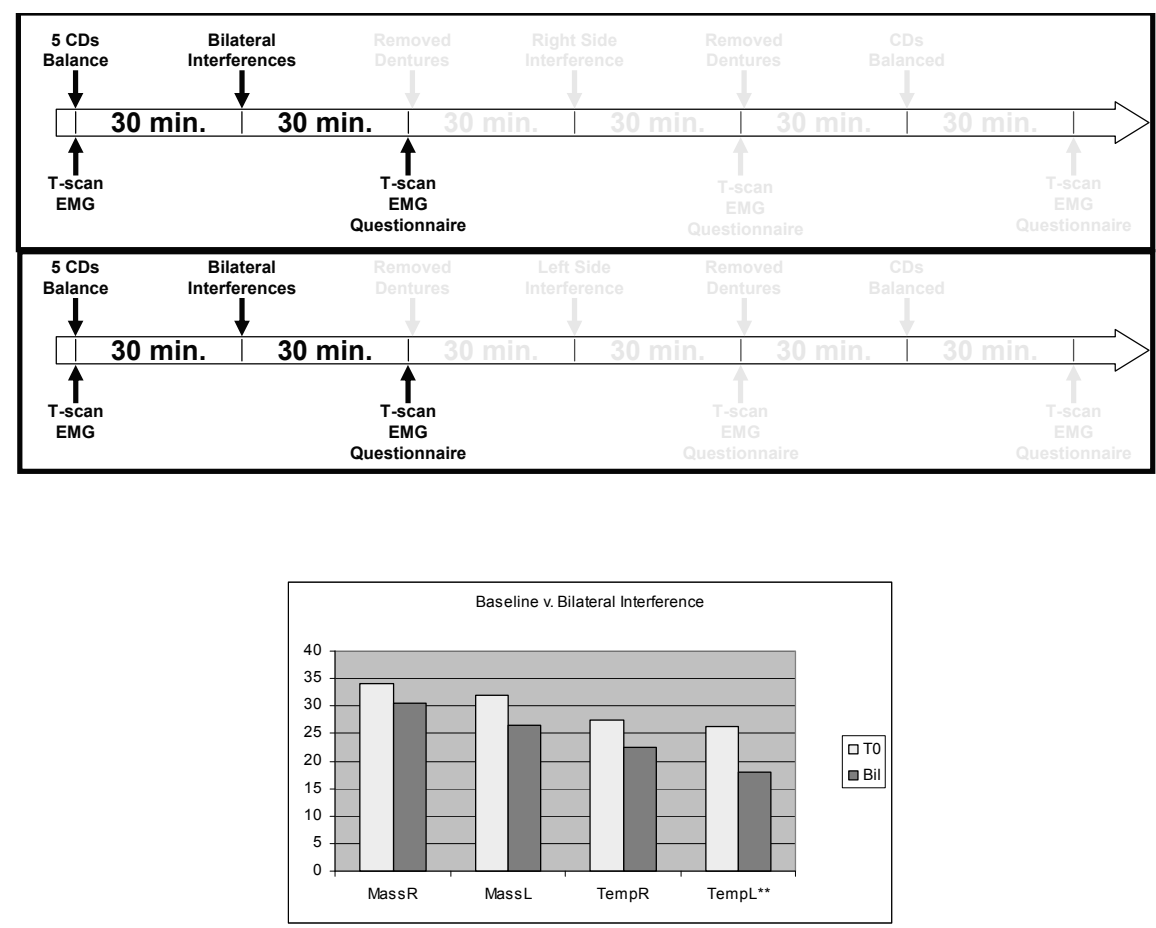

Figure 10 Timeline and the graphical analysis of the EMG activity at baseline (T0) and EMG activity after the introduction of the bilateral interferences (Bil) in all subjects

(Mass R-Right Masseter, Mass L-Left Masseter, Temp R-Right anterior Temporalis, Temp L-Left anterior Temporalis)

Note: At significance level of .05 no significant difference was found between the two. 

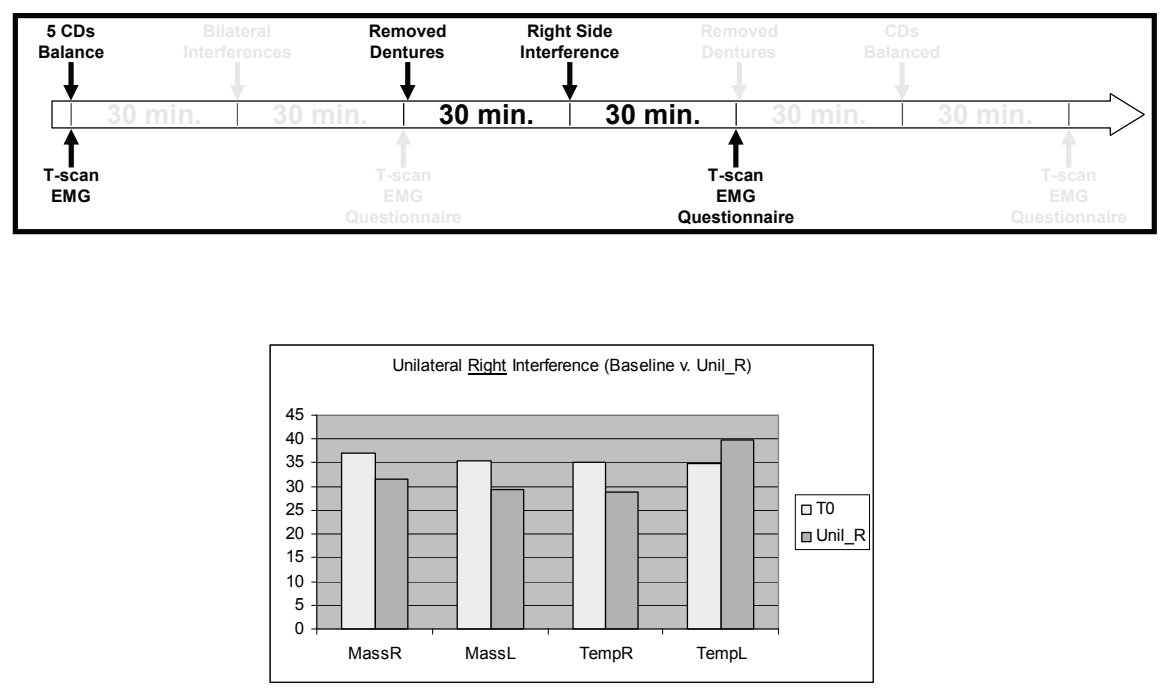

Figure 11 Timeline and the graphical analysis of the EMG activity at baseline (T0) and EMG activity after the introduction of the right interferences (Unil_R) in five subjects

(Mass R-Right Masseter, Mass L-Left Masseter, Temp R-Right anterior Temporalis, Temp L-Left anterior Temporalis)

Note: At significance level of .05 no significant difference was found between the two. 

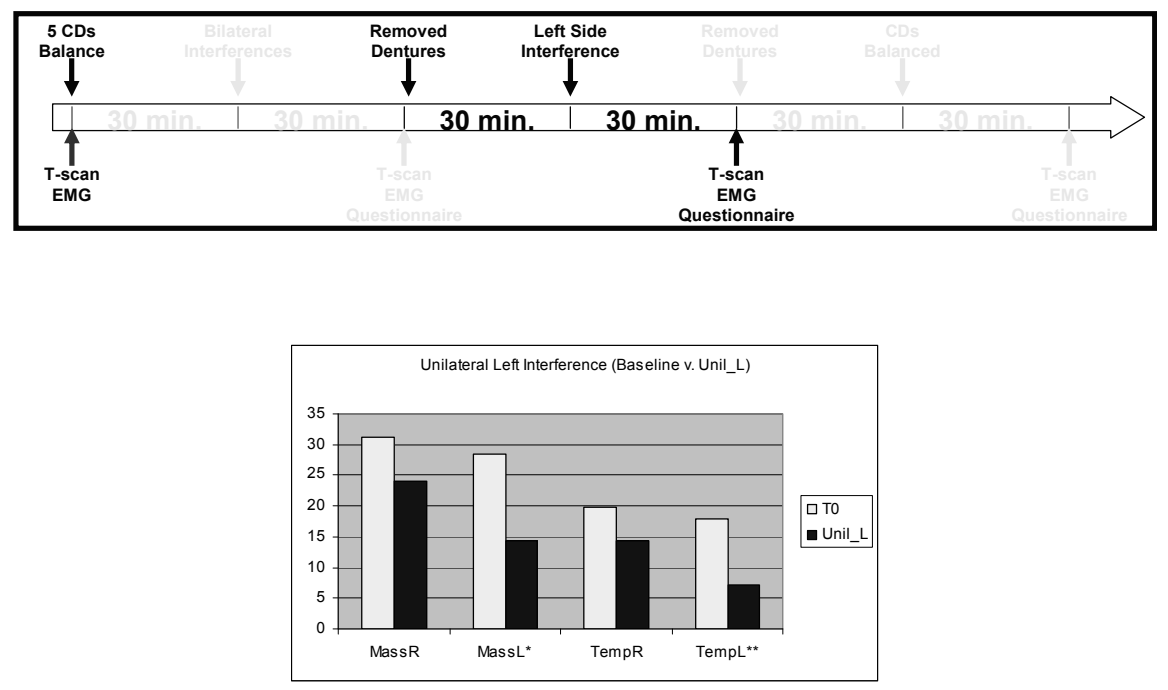

Figure 12 Timeline and the graphical analysis of the EMG activity at baseline (T0) and EMG activity after the introduction of the left interferences (Unil_L) in five subjects

(Mass R-Right Masseter, Mass L-Left Masseter, Temp R-Right anterior Temporalis, Temp L-Left anterior Temporalis)

Note: At significance level of .05 a significant difference was found between the left masseter activities. 
(3) Comparison of baseline EMG and EMG after elimination of interferences:

- When the EMG activity at baseline was compared with the EMG activity after the elimination of the unilateral interferences in five subjects who had right sided unilateral interference at significance level of .05 no significant difference was found between the two, see Figure 13.

- When the EMG activity at baseline was compared with the EMG activity after the elimination of the unilateral interferences in five subjects who received left sided unilateral interference at significance level of .05 no significant difference was found between the two, see Figure 14.

- When the EMG activity at baseline was compared with the EMG activity after the elimination of the unilateral interferences in all subjects at significance level of .05 no significant difference was found between the two, see Figure 15. The analysis of questionnaire did not reveal any significant finding. The subjects did not perceive any change in their bite. 

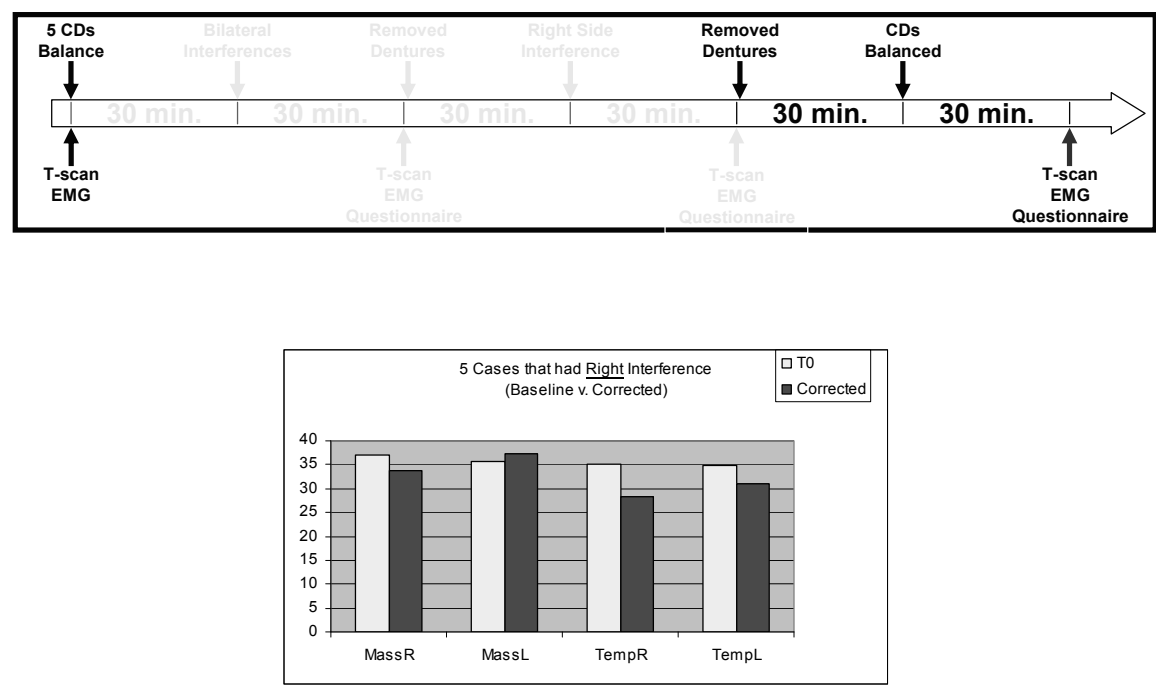

Figure 13 Timeline and the graphical analysis of the EMG activity at baseline (T0) and EMG activity after the elimination of the interferences (corrected) of five subjects who received right sided unilateral interference

(Mass R-Right Masseter, Mass L-Left Masseter, Temp R-Right anterior Temporalis, Temp L-Left anterior Temporalis)

Note: At significance level of .05 no significant difference was found between the two. 

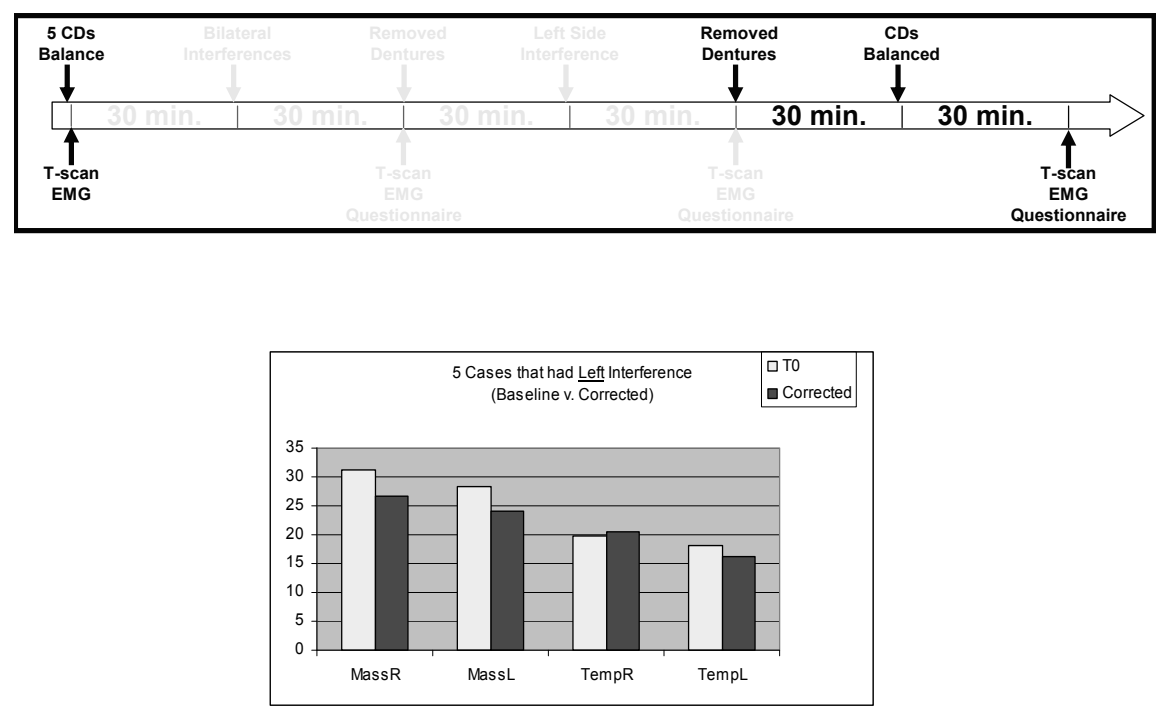

Figure 14 Timeline and the graphical analysis of the EMG activity at baseline (T0) and EMG activity after the elimination of the interferences (corrected) in five subjects who received left sided unilateral interference

(Mass R-Right Masseter, Mass L-Left Masseter, Temp R-Right anterior Temporalis, Temp L-Left anterior Temporalis)

Note: At significance level of .05 no significant difference was found between the two. 

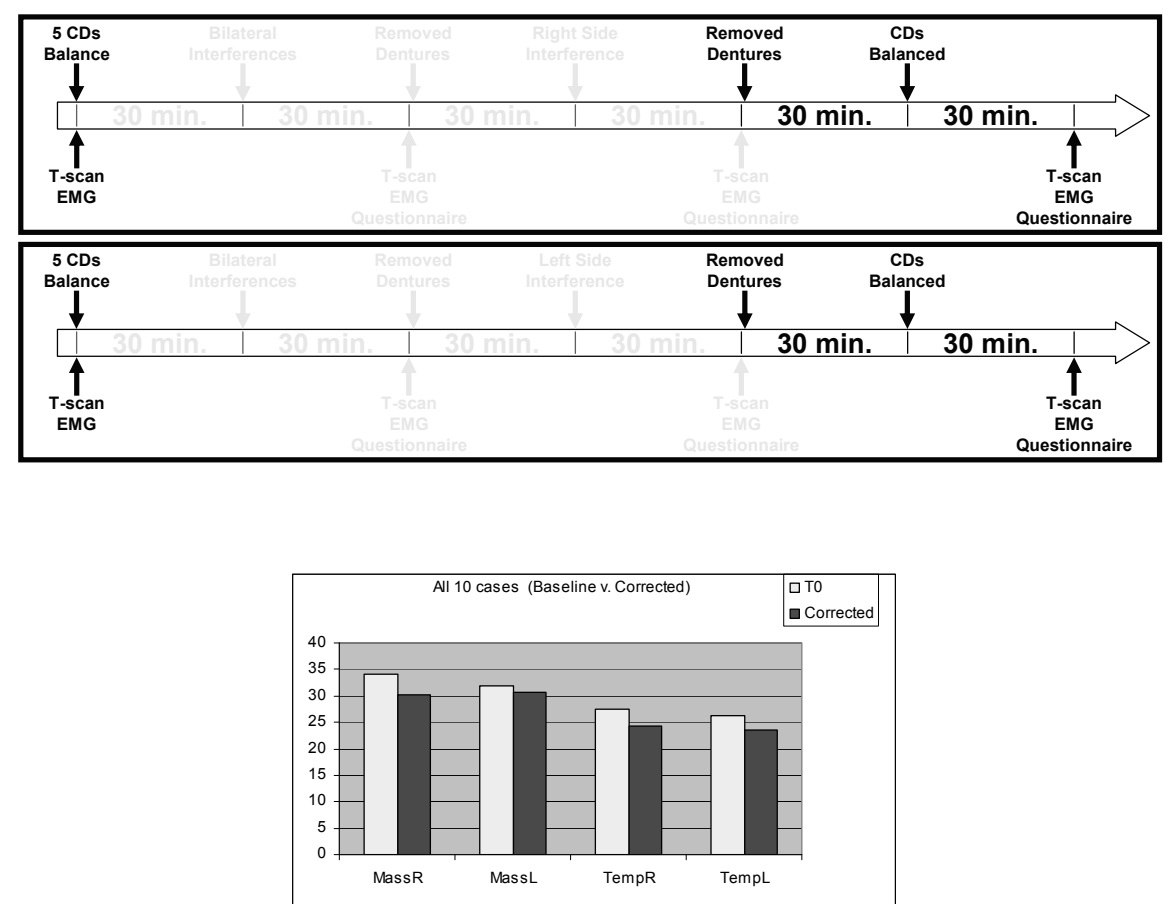

Figure 15 Timeline and the graphical analysis of the EMG activity at baseline (T0) and EMG activity after the elimination of the interferences (corrected) in all subjects

(Mass R-Right Masseter, Mass L-Left Masseter, Temp R-Right anterior Temporalis, Temp L-Left anterior Temporalis)

Note: At significance level of .05 no significant difference was found between the two. 


\section{CHAPTER 6. DISCUSSION}

Studies on dentate subjects have shown that maximum bilateral occlusal stability is a prerequisite for optimum neuromuscular generation of well adjusted bilateral clenching forces which in turn appears to facilitate bilateral central motor commands of equal strength. On the other hand unilateral occlusal instability seems to distort the commands to the paired elevator muscles (Bakke \& Moller, 1980).

The objective of complete denture occlusion is to direct an even transfer of occlusal forces to the denture supporting tissues. Failure to accomplish the same can lead to unstable dentures, subject dissatisfaction and sore spots (Olivieri, 1998). The masseter muscle activity of the edentulous individual is significantly lower than a dentate individual which is a result of the instability of the lower denture requiring continuous control of the dynamic mandibular posture (Fontijn, 2000; Veyrune, 2000).

The results of the present study suggest that there was no significant difference in the EMG values of the subjects upon introduction of the bilateral interferences. Also there was no significant difference in the EMG values of subjects upon introduction of unilateral right interferences.

In the five cases with unilateral left interferences there was a significant reduction in the EMG activity of the left masseter. The decrease in muscle activity could be an avoidance behavior to protect the surrounding oral and perioral structures (Michelotti, 2005). The new intra oral stimuli due to the occlusal interferences may have activated the nociceptive afferents which inhibited muscle contraction as a protective reflex, as was also observed by Nergiz (1992) and Sohn (2000) in their study.

There was also no significant difference in the EMG values at baseline and after the removal of the interferences. The analysis of the questionnaires did not reveal any significant finding. The subjects investigated in the present study were healthy physiologically and psychologically. Psychologically distressed subjects might react differently to the introduction of an occlusal disturbance (Michelotti, 2005).

To the author's knowledge, this is the first short term study investigating the effect of occlusal interference on the EMG activity of the masseter and temporalis on edentulous subjects; therefore, the findings cannot be directly compared with those of previous studies. This should be considered a pilot study and the results of this study should be interpreted with caution owing to the small sample size and the variability in the subject population. Adaptation of the neuromuscular system takes a long time and may be a determinant factor in influencing EMG activity and this aspect can change the results (Raustia, 1996).

The small sample size of ten subjects is a limitation of this study. Also investigation for a longer duration on the effect of the occlusal interference will get more convictive results. The limitation of the surface EMG recording cannot be overlooked 
even though methods were used to control the influence of interferential factors for example maintaining the same positions of the electrodes in different recordings. But the gender or inter-individual differences in the EMG activity did not control well. 


\section{CHAPTER 7. CONCLUSION}

The results of the present study suggest:

(1) There was no significant difference in the EMG values of the subjects after the introduction of the bilateral interferences from the baseline and the analysis of the questionnaires after the introduction of bilateral interferences did not reveal any significant finding.

(2) There was no significant difference in the EMG values of the subjects after the introduction of the unilateral right interferences from the baseline and the analysis of the questionnaires after the introduction of the unilateral right interference did not reveal any significant finding.

(3) In the five cases with unilateral left interferences there was a significant reduction in the EMG activity of the left masseter as compared to the baseline but the analysis of the questionnaires after the introduction of the unilateral left interference did not reveal any significant finding. The new intra oral stimuli may have activated the nociceptive afferents which inhibited muscle contraction as a protective reflex (Nergiz, 1992 and Sohn, 2000).

(4) There was also no significant difference in the EMG values at baseline and after the removal of the interferences. The questionnaires after the removal of the interference did not reveal any significant finding. 


\section{LIST OF REFERENCES}

Bakke M., Moller E. Distortion of maximal elevator activity by unilateral premature tooth contact. Scand J Dent Res. 1980; 88: 67-75.

Bhatka R., Throckmorton G.S., Wintergerst A.M., Hutchins B., Buschang P.H. Bolus size and unilateral chewing cycle kinematics. Arch Oral Biol. 2004; 49: 559-66.

Boening K. W., Walter M.H. Computer aided evaluation of occlusal load in complete dentures. J Prosthet Dent. 1992; 67: 339-44

Christensen L.V., Rassouli N.M. Experimental occlusal interferences. Part II. Masseteric EMG responses to an intercuspal interference. J Oral Rehabil. 1995; 22: $521-31$.

Finbarr P., McMillan A., Walshaw D. A subject based assessment of implant stabilized and conventional complete dentures. J Prosthet Dent. 2001; 85: 141-7.

Fontijn-Tekamp F.A., Slagter A.P., van der Bilt A., van't Hof M.A., Witter D.J., Kalk W., Jansen J.A. Biting and chewing in overdentures, full dentures, and natural dentitions. J Dent Res. 2000; 79: 1519-24.

Garett N.R., Kaurich M., Perez P., Kapur K.K. Masseter muscle activity in denture wearers with superior and poor masticatory performance. J Prosthet Dent. 1995; 74 : 628-36.

Garett N.R., Kapur K.K. and Perez P. Effects of improvements of poorly fitting dentures and new dentures on subject satisfaction. J Prosthet Dent. 1996; 76: 403-13.

Garett N.R., Perez P., Elbert C., Kapur K.K. Effects of improvements of poorly fitting dentures and new dentures on masseter activity during chewing. J Prosthet Dent. 1996; 76: 394-402.

Hugger A., Hugger S., Schindler H.J. Surface electromyography of the masticatory muscles for application in dental practice. Current evidence and future developments. Int J Comput Dent. 2008; 11: 81-106.

Ingervall B., Carlsson G. Masticatory muscle activity before and after elimination of balancing side occlusal interference. J Oral Rehabil. 1982; 9: 183-92.

Li J., Wang T., Zhang Z., IshikawaT. The electromyographic activity of masseter and anterior temporalis during orofacial symptoms induced by experimental high spot. J Oral Rehabil. 2007; 35: 79-87. 
Kapur K.K., Garett N.R. Studies of biologic parameters for denture design. Part II: Comparison of masseter muscle activity, masticatory performance, and salivary secretion rates between denture and natural dentition groups. J Prosthet Dent. 1984; 52: 408-13.

Kapur K.K., Soman S.D. Masticatory performance and efficiency in denture wearers. J Prosthet Dent. 2006; 95: 407-11.

Kerstein R. Obtaining measurable bilateral simultaneous occlusal contacts with computer analyzed and guided occlusal adjustments. Quintessence Int. 2001; 32: 7-18

Keubker W.A. Denture problems: causes, diagnostic procedures, and clinical treatment. Part I. Quintessence Int. 1984; 10: 1031-4.

Keubker W.A. Denture problems: causes, diagnostic procedures, and clinical treatment. Part II. Quintessence Int. 1984; 11: 1131-41.

Makofsky H. The influence of forward head posture on dental occlusion. J Craniomand Pract. 2000; 18: 30-39.

Maness W., Benjamin M., Podoloff R., Bobbick A., Golden R. Computerized occlusal analysis. A new technology. Quintessence Int. 1987; 4: 287-92.

McHorris W.H. Occlusion with particular emphasis on the functional and parafunctional role of anterior teeth. Part 1. J Clin Orthod. 1979; 13: 606-20.

Michelotti A., Farella M., Gallo L., Veltri A., Palla S. and Martina R. Effect of Occlusal Interference on Habitual Activity of Human Masseter. J Dent Res. 2005; 84: 644-48.

Moyers R.E. Temporomandibular muscle contraction patterns in angle class II division 1 malocclusions: an electromyographic analysis. Am J Orthod. 1949; 35: 837-57.

National Institute of Dental and Craniofacial Research. Oral health of United States adults: regional findings. Bethesda, MD: NIDCR 1988.

Nergiz I., Proschel P., Niedermeier W. Incorporation and occlusal stability of complete dentures. Dtsch Zahnarztl Z. 1992; 47: 818-21.

Olivieri F., Kang K.H., Hirayama H., Maness W.L. New Method for analyzing complete denture occlusion using center of force concept: A clinical report. J Prosthet Dent. 1998; 80: 519-23.

Perez P., Kapur K.K., Garret N. Studies of biologic parameters for denture design Part III: Effects of occlusal adjustment, base retention, and fit on masseter muscle activity and masticatory performance. J Prosthet Dent. 1985; 53: 69-73. 
Piancino M., Farina D., Talpone F., Castroflorio T., Gassino G., Margarino V., Bracco P. Surface EMG of jaw-elevator muscles and chewing pattern in complete denture wearers. J Oral Rehabil. 2005: 32; 863-70.

Pruzansky S. The application of electromyography to dental research. J Am Dent Assoc 1952; 44: 49-68.

Raustia A.M., Salonen M.A., Pyhtinen J. Evaluation of masticatory muscles of edentulous subjects by computed tomography and electromyography. J Oral Rehabil. 1996; 23: 11-6.

Riise C., Sheikholeslam A. The influence of experimental interfering occlusal contacts on the postural activity of the anterior temporal and masseter muscles in young adults. J Oral Rehabil. 1982; 9: 419-25.

Tallgren A., Lang B.R., Holden S., Miller R.L. Longitudinal electromyographic study of swallowing patterns in complete denture wearers. Int J Prosthodont. 1995; 8: 46778.

Todd J.E., Lader D. Adult dental health 1988 United Kingdom. London: HMSO 1991.

Sohn M.K., Gravenielsen T., Arendt Nielsen L., Svensson P. Inhibition of motor unit firing during experimental muscle pain in humans. Muscle Nerve. 2000; 23: 1219-26.

Veyrune J.L., Mioche L. Complete denture wearers: EMG of mastication and texture perception whilst eating meat. Eur J Oral Sci. 2000; 108: 83-92.

Zarb G.A. The edentulous milieu. J Prosthet Dent. 1983; 49: 825-31. 


\section{APPENDIX A. QUESTIONS AND EXAMPLE OF RESPONSE CHOICES FOR SUBJECT ASSESMENT OF STUDY DENTURES AFTER THE INTRODUCTION OF OCCLUSAL INTERFERENCES}

Subject Name:

ID \#:

Examiner:

Date:

Question 1: Compared to the first time the complete denture was placed in your mouth does the bite feel accurate?
a. Yes
b. No

Question 2: If the bite feels inaccurate, can you point out where is it high?
a. Front
b. Back
c. Left
d. Right
e. No perceivable inaccuracy

Question 3: If the bite feels inaccurate, do you believe it will affect your speech?
a. Yes
b. No

Question 4: If the bite feels inaccurate, do you believe it will affect your ability to chew?
a. Yes
b. No

Question 5: Do you believe you can adapt to the inaccuracy of your existing bite?
a. Yes
b. No

Question 6: Do you believe your existing bite will cause pain?
a. Yes
b. No 


\section{APPENDIX B. QUESTIONS AND EXAMPLE OF RESPONSE CHOICES FOR SUBJECT ASSESMENT OF STUDY DENTURES AFTER THE ELIMINATION OF OCCLUSAL INTERFERENCES}

Subject Name:

ID \#:

Examiner:

Date:

Question 1: Compared to the first time the complete denture was placed in your mouth does the bite feel accurate?
a. Yes
b. No
c. I am not sure

Question 2: If the bite feels inaccurate, can you point out where is it high?
a. Front
b. Back
c. Left
d. Right
e. No perceivable inaccuracy

Question 3: If the bite feels accurate, do you believe it will improve your speech?
a. Yes

b. No

Question 4: If the bite feels accurate, do you believe it will improve your ability to chew?
a. Yes

b. No

Question 5: Do you believe you can adapt to your existing bite?
a. Yes
b. No

Question 6: Do you believe your existing bite will cause pain?
a. Yes
b. No 


\section{VITA}

Dr. Swati Ahuja was born on September 23, 1980. She received her Bachelor in Dental Science (BDS) degree from the Nair Hospital Dental College in 2002. She was accepted into the University of Tennessee Health Science Center Advanced Prosthodontic program. Dr. Ahuja is currently a third year resident, specializing in fixed and removable prosthodontics at UTHSC. She is also working towards her Master of Dental Science degree from the University of Tennessee. She is a current member of the American College of Prosthodontists and the American Dental Association. 